\title{
Transport in magnetically doped topological insulators: Effects of magnetic clusters
}

\author{
A. N. Zarezad and J. Abouie* \\ Department of Physics, Institute for Advanced Studies in Basic Sciences (IASBS), Zanjan 45137-66731, Iran
}

(Dated: October 17, 2018)

\begin{abstract}
We study the electron transport in a magnetically doped three dimensional topological insulator (TI) by taking the effects of impurity-impurity exchange interactions into account. The interactions between magnetic impurities give rise to the formation of magnetic clusters with temperature dependent mean sizes, randomly distributed on the surface of the TI. Instead of dealing with single magnetic impurities, we consider surface Dirac electrons to be scattered off magnetic clusters, and define the scattering potential in terms of clusters mean sizes. Within the semiclassical Boltzmann approach, employing a generalized relaxation time approximation, we obtain the surface conductivity of the TI by solving four sets of recursive relations and demonstrate that, the system is highly anisotropic and the surface conductivities possess non-monotonic behaviors, they strongly depends on the direction, the mean size and the number of magnetic clusters. We demonstrate that the dependence of the anisotropic magnetoresistance (AMR) to the spin direction of the magnetic clusters is inconsistent with the angular dependence of the TI doped with non-interacting magnetic impurities. Our results are consistent with the recent experiment on the AMR of the Cr-doped $(\mathrm{Bi}, \mathrm{Sb})_{2} \mathrm{Te}_{3} \mathrm{TI}$.
\end{abstract}

\section{INTRODUCTION}

Topological insulators (TIs) are a new class of materials which attracted intensive theoretical ${ }^{1-4}$ and experimental ${ }^{5,6}$ attentions in condensed matter physics. TIs are band insulators in the bulk while conducting along the surfaces, hosting metallic surface states with a Dirac-cone-like dispersion. The Dirac cone is located in the center of the Brillouin zone and the spin and momentum degrees of freedom are locked. The spin-momentum locking of surface Dirac electrons is protected by timereversal symmetry ${ }^{1,2,7-10}$, which leads to a variety of interesting effects ${ }^{11-13}$, in particular to the suppression of the elastic backscattering of surface states in the absence of spin-flip processes ${ }^{14,15}$. The robustness of these topologically protected processes, when introducing impurities which break time-reversal symmetry, is of critical importance for spin-based transport in such materials.

Recently, many efforts have been devoted to the investigation of the surface transport properties of $3 \mathrm{D}$ TIs in the presence of localized and identical magnetic impurities ${ }^{16-19}$. In dilute magnetic TIs, the impurities are uncorrelated and distributed randomly on the surface of the TI, thus single-impurity-scattering processes (the scattering of the massless Dirac electrons by a single magnetic impurity), specify the behavior of the surface resistivity. In this case, the scattering amplitudes and consequently the surface magneto-resistance strongly depend on the orientation of impurities' spins ${ }^{15,20,21}$. By increasing doping the interactions of magnetic impurities become important and their effects on the transport properties of the TI are crucial. The multiple-impurityscattering problem of the massless Dirac electrons in the presence of magnetic impurities normal to the surface of the TI has been evaluated theoretically in Ref. ${ }^{22}$. It has been shown that, different from the single-impurityscattering, the Hall component of resistivity and the in- verse momentum relaxation time exhibit oscillatory behavior due to the interference during the double- and triple-impurities-scattering processes. In spite of many studies on the electron transport of magnetic TIs, the effects of magnetic impurity interactions on the magnetoresistance of TIs have not been addressed so far.

In this paper, we investigate the effects of magnetic impurity-magnetic impurity exchange interactions on the transport in a magnetic TI. We consider a 3D TI doped with magnetic impurities localized on the surface of the TI, and investigate the surface magneto-resistance of the TI by taking the exchange interactions of magnetic impurities into account. The exchange interactions of magnetic impurities give rise to the formation of "magnetic clusters", constructed of correlated magnetic impurities with the same direction. We consider that the surface massless Dirac electrons scatter by magnetic clusters rather than single impurities. The cluster concept was first introduced in Refs. ${ }^{23,24}$ to explain the temperature dependence of magneto-resistance of magnetic semiconductors. The clusters' mean size (CMS) and the number of clusters with mean sizes $(\mathrm{CN})$ depend on temperature, they increase by increasing temperature, pass through a maximum at a critical temperature, and decrease toward zero at high temperatures ${ }^{23,24}$. We model the scattering of massless Dirac electrons off magnetic clusters with a potential, exponentially decaying with the electron-cluster separation distance rescaled by the CMS. In order to obtain the surface conductivity of such a system we employ the semi-classical Boltzmann approach. However, since the scattering of Dirac electrons by a magnetic cluster is not isotropic, the standard relaxation time approximation is not applicable here. Employing a generalized relaxation time approximation ${ }^{25}$ and solving four sets of recursive relations we obtain the relaxation times of Dirac electrons and demonstrate that, the system is highly anisotropic and the surface conductivity strongly 
depends on the CMS, the CN and the spin directions of magnetic clusters. We show that surface conductivities possess a non-monotonic behavior, in general they decrease sharply by increasing CMS and pass through a minimum where the electrons Fermi wavelength is identically the same as CMS. We demonstrate that the angular dependence of the AMR is inconsistent with the angular dependence of the TI doped with non-interacting magnetic impurities. We also show that our results are consistent with the recent experiment on the AMR of the Cr-doped (Bi, Sb) ${ }_{2} \mathrm{Te}_{3} \mathrm{TI}^{26}$

The outline of this paper is as follows. In Sec. II, we introduce our model and present the scattering potential in terms of CMS. In Sec. III, we obtain the transition amplitudes of the system. Employing a generalized relaxation time approximation we give a closed form for the effective relaxation times of massless Dirac electrons. In Sec. IV, we compute the surface conductivities of the TI, both in isotropic and anisotropic cases. In this section we investigate, in detail, the behavior of the surface conductivities for different values of CMS and clusters' spin directions. We also investigate the angular dependence of the AMR for different values of CMS. In Sec. V, we also obtain the surface conductivity of a 3D TI doped with non-magnetic impurities and compare the behaviors of the relaxation times in both systems. We finally summarize our results in Sec. VI and give the concluding remarks.

\section{OUR MODEL}

We consider a 3D topological insulator (TI) which its surface defines the $x y$ plane and the surface is normal to $z$ direction. The minimal Hamiltonian of the TI describing the dynamics of the surface electrons, is given $b y^{27,28}$ :

$$
H_{0}=\hbar v_{\mathrm{F}}(\mathbf{k} \times \boldsymbol{\sigma})_{z},
$$

where $v_{\mathrm{F}}$ in the Fermi velocity, $\mathbf{k}=\left(k_{x}, k_{y}\right)$ is the vector of momentum and $\boldsymbol{\sigma}$ is the vector of Pauli matrices, describing the electron spin. The eigenvalues and eigenvectors of the Hamiltonian $H_{0}$ are given by:

$$
\varepsilon^{ \pm}= \pm \hbar v_{\mathrm{F}} k
$$

and

$$
\psi_{\mathbf{k}, \pm}(\mathbf{r})=\frac{e^{i \mathbf{k} \cdot \mathbf{r}}}{\sqrt{2 A}}\left(\begin{array}{c}
\mp i \mathrm{e}^{-i \phi} \\
1
\end{array}\right)
$$

where $\hbar$ is the Planck constant divided by $2 \pi, A$ is the TI surface area, $k=|\mathbf{k}|=\left(k_{x}^{2}+k_{y}^{2}\right)^{1 / 2}$ is the modulus of the electron momentum, and $\phi=\arctan \left(k_{y} / k_{x}\right)$ is the polar angle between the momentum vector and the $k_{x}$ direction. It is important to note that the spin states (3) are always perpendicular to the motion direction.

In TIs, doped with magnetic impurities, the interaction of impurities with Dirac electrons affects the transport properties of the TI. In dilute magnetic TI, the exchange interactions between magnetic impurities (MI-MI

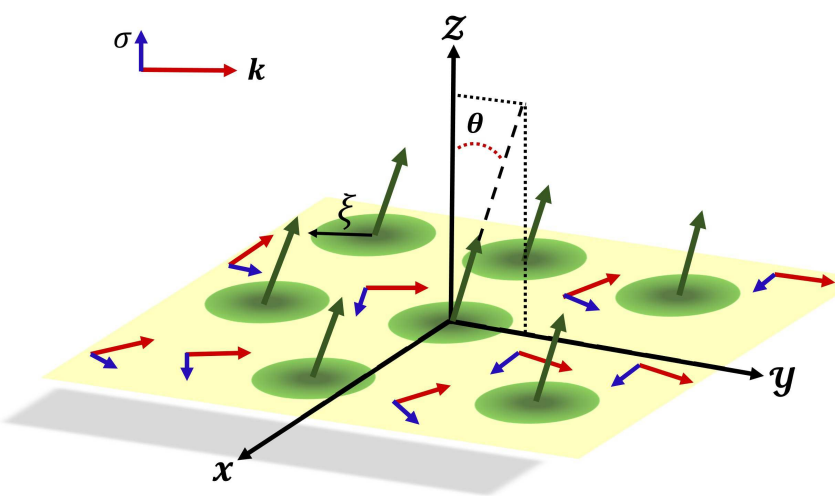

FIG. 1. (Color online) The schematic illustration of the surface of a TI with randomly distributed magnetic clusters (circles). $\xi$ is CMS, green arrows show the spins of clusters, and $\theta$ is their tilt angle with $z$ axis. The red and blue arrows show the momentum and spin of Dirac electrons, respectively.

interactions) are negligible and we can ignore their effects on the surface conductivity of the TI. By increasing doping, the MI-MI exchange interactions become considerable and we should take them into account in the spin dependent transport of the TI. The presence of MI-MI exchange interactions, gives rise to the formation of magnetic clusters with different sizes in the entire system. There are two rules for constructing magnetic clusters in a spin system ${ }^{29}$, one is due to purely geometric effects which makes cluster's spins to be in the same direction and the other is due to the correlation between the cluster's spins. Considering such a definition of cluster, Coniglio and Klein ${ }^{30}$ showed that by throwing bonds between nearest-neighbor pairs of parallel spins with a probability: $p=1-\exp \left(-2 J_{I} / k_{\mathrm{B}} T\right)$, the spins of the system can be divided into clusters made of parallel spins connected by bonds. Here, $J_{I}$ is the nearest neighbor exchange interaction between impurities and $k_{\mathrm{B}}$ is Boltzmann constant. The number of clusters and their mean sizes could be computed numerically by using cluster counting algorithms, like the Hoshen-Kopelman algorithm ${ }^{31}$, one of the most popular cluster identification algorithm. The number of clusters and their mean sizes depend on temperature. In general, they increase by temperature, pass through a maximum around the critical temperature of the surface (neglecting the back-action effects of Dirac electrons on the formation of magnetic cluster) and decrease toward zero at high temperatures.

In order to study the effects of MI-MI exchange interactions on the behavior of surface conductivity, we consider magnetic clusters as scattering centers and suppose that Dirac electrons interact with these centers rather than single impurities ${ }^{23,24}$. By defining the parameter $\xi$ as the mean size of magnetic clusters, we model the interaction of a Dirac electron located at $\mathbf{r}$ with a cluster centered at $\mathbf{R}$ as:

$$
H_{\sigma S}=J(\mathbf{r}-\mathbf{R}) \boldsymbol{\sigma}(\mathbf{r}) \cdot \mathbf{S}(\mathbf{R})
$$


where

$$
J(\mathbf{r}-\mathbf{R})=J_{0} \exp (-|\mathbf{r}-\mathbf{R}| / \xi) .
$$

Here, $\mathbf{S}$ is the spin of magnetic clusters and $J_{0}$ is coupling constant. The scattering potential in Eq. (4) is long-range and the CMS $\xi$ is served as the range of scattering potential. Magnetic clusters are uncorrelated and distributed randomly on the surface of the TI. Thus, we can investigate their effects on the surface conductivity of the TI, separately. Without loss of generality we suppose that clusters' spins lie on $y z$ plane, i.e. $\mathbf{S}=S(0, \sin \theta, \cos \theta)$, where $\theta$ is the tilt angle between $\mathrm{S}$-vector and $z$ axis (see the schematic Fig. 1).

\section{ANISOTROPIC RELAXATION TIMES}

In order to obtain the surface conductivity of the magnetic TI we use semiclassical Boltzmann formalism. In the presence of an applied electric field $\mathbf{E}$, the nonequilibrium distribution function $f$ depends on the electrons' k-vector and the electric field, satisfying the following relation ${ }^{32}$ :

$$
\frac{\partial f}{\partial t}=e \mathbf{v}_{\mathbf{k}} \cdot \mathbf{E}\left(\frac{\partial f^{0}}{\partial \varepsilon}\right)
$$

where $e<0, \mathbf{v}_{\mathbf{k}}=v_{\mathbf{k}}(\cos \phi$, $\sin \phi)$ is the velocity of Dirac electrons and $f^{0}=f^{0}(\varepsilon)$ is the Fermi-Dirac distribution function. Considering elastic scattering, and using detailed balance $\partial f / \partial t$ can also be written as:

$$
\frac{\partial f}{\partial t}=A \int \frac{d^{2} k^{\prime}}{(2 \pi)^{2}} w\left(\mathbf{k}, \mathbf{k}^{\prime}\right)\left[f(\mathbf{k}, \mathbf{E})-f\left(\mathbf{k}^{\prime}, \mathbf{E}\right)\right],
$$

where $w\left(\mathbf{k}, \mathbf{k}^{\prime}\right)$ is transition rate between the two eigenstates $\left(|\mathbf{k}\rangle\right.$ and $\left.\left|\mathbf{k}^{\prime}\right\rangle\right)$ of the Dirac Hamiltonian $H_{0}$. Using Fermi golden rule, the transition rate is written in terms of the scattering amplitude $\left|T_{\mathbf{k}, \mathbf{k}^{\prime}}\right|^{2}$ as follows:

$$
w\left(\mathbf{k}, \mathbf{k}^{\prime}\right)=\frac{2 \pi}{\hbar}\left|T_{\mathbf{k}, \mathbf{k}^{\prime}}\right|^{2} \delta\left(\varepsilon_{k}-\varepsilon_{k^{\prime}}\right) .
$$

Within the first Born approximation, the $T$-matrix is given by: $T_{\mathbf{k}, \mathbf{k}^{\prime}} \approx\left\langle\mathbf{k}\left|\mathcal{H}_{\sigma S}\right| \mathbf{k}^{\prime}\right\rangle$, where $\mathcal{H}_{\sigma S}$ is the scattering Hamiltonian given by $\mathcal{H}_{\sigma S}=\sum_{\mathbf{r}, \mathbf{R}} H_{\sigma S}$. Since magnetic clusters are uncorrelated and distributed randomly on the surface of the TI, we can write the transition rate as $w\left(\mathbf{k}, \mathbf{k}^{\prime}\right)=\frac{2 \pi}{\hbar} n_{c}\left|T_{\mathbf{k}, \mathbf{k}^{\prime}}\right|^{2} \delta\left(\varepsilon_{k}-\varepsilon_{k^{\prime}}\right)$, where $n_{c}$ is CN. By defining the parameter $\tau_{\mathbf{k}}$ as the momentum relaxation time of electrons and using the relaxation time approximation: $-\left(f-f^{0}\right) / \tau=\partial f / \partial t$, the non-equilibrium distribution function is given by:

$$
f=f^{0}+e \tau_{\mathbf{k}} \mathbf{v}_{\mathbf{k}} \cdot \mathbf{E}\left(\frac{\partial f^{0}}{\partial \varepsilon}\right) .
$$

Making use of Eqs. (6), (7) and (9) we readily obtain:

$$
\mathbf{v}_{\mathbf{k}} \cdot \mathbf{E}=A \int \frac{d^{2} k^{\prime}}{(2 \pi)^{2}} w\left(\mathbf{k}, \mathbf{k}^{\prime}\right)\left(\tau_{\mathbf{k}} \mathbf{v}_{\mathbf{k}} \cdot \mathbf{E}-\tau_{\mathbf{k}^{\prime}} \mathbf{v}_{\mathbf{k}^{\prime}} \cdot \mathbf{E}\right)
$$

When the system is isotropic, (in the case of $\theta=0$ ), the scattering amplitude depends on the angle of $\mathbf{k}$ and $\mathbf{k}^{\prime}$ and hence the relaxation time depends only on the magnitude of $\mathbf{k}$ and $\mathbf{k}^{\prime}$. Since $k=k^{\prime}$, Eq. (10) is simplified and the relaxation time is given by the standard formula ${ }^{32}$ : $\frac{1}{\tau_{k}}=A \int \frac{d^{2} k^{\prime}}{(2 \pi)^{2}} w\left(\mathbf{k}, \mathbf{k}^{\prime}\right)[1-\cos \Delta \phi]$, where $\Delta \phi=\phi-\phi^{\prime}$, with $\phi=\arctan \left(k_{y} / k_{x}\right)$ and $\phi^{\prime}=\arctan \left(k_{y}^{\prime} / k_{x}^{\prime}\right)$. In the case of $\theta \neq 0$, the system is anisotropic and the scattering amplitude as well as the relaxation time depend on both the magnitude and directions of $\mathbf{k}$ and $\mathbf{k}^{\prime}$, and consequently the standard scheme is not appropriate anymore.

The transport properties of anisotropic systems have been studied using different approaches ${ }^{25,33-35}$. In this paper, we employ the recipe presented in Ref. 25, and approximate the non-equilibrium distribution function, to linear order in electric field, as:

$$
f-f^{0}=e E v_{\mathbf{k}}\left(\frac{\partial f^{0}}{\partial \varepsilon}\right)[a(\phi) \cos \chi+b(\phi) \sin \chi],
$$

where $\chi$ is the angle between electric field and $x$ axis. Here, the two independent functions $a(\phi)$ and $b(\phi)$ have a dimension of time. By substituting Eq. (11) into Eq. (7) and using Eq. (6) we achieve the following two decoupled inhomogeneous Fredholm equations:

$$
\begin{aligned}
\cos \phi & =\bar{w}(\phi) a(\phi)-\int d \phi^{\prime} w\left(\phi, \phi^{\prime}\right) a\left(\phi^{\prime}\right), \\
\sin \phi & =\bar{w}(\phi) b(\phi)-\int d \phi^{\prime} w\left(\phi, \phi^{\prime}\right) b\left(\phi^{\prime}\right),
\end{aligned}
$$

where $\bar{w}(\phi)=\int d \phi^{\prime} w\left(\phi, \phi^{\prime}\right)$ and $w\left(\phi, \phi^{\prime}\right)=$ $\frac{A}{(2 \pi)^{2}} \int k^{\prime} d k^{\prime} w\left(\mathbf{k}, \mathbf{k}^{\prime}\right)$. For solving these equations and obtaining the non-equilibrium distribution function, we look for a solution in the form of Fourier series:

$$
\begin{gathered}
a(\phi)=a_{0}+\sum_{n=1}\left(a_{n}^{c} \cos n \phi+a_{n}^{s} \sin n \phi\right), \\
b(\phi)=b_{0}+\sum_{n=1}\left(b_{n}^{c} \cos n \phi+b_{n}^{s} \sin n \phi\right) .
\end{gathered}
$$

Putting the Fourier expansions of $a(\phi)$ and $b(\phi)$ into Eqs. (12) and (13), we reach four sets of linear recursive relations between the coefficients $a_{n}^{c}, a_{n}^{s}, b_{n}^{c}$ and $b_{n}^{s}$. Depending on the function $w\left(\phi, \phi^{\prime}\right)$, the solutions are exact or reasonably approximate.

Due to the elastic scattering of Dirac electrons from magnetic clusters, the inter-band transitions are forbidden and intra-band transitions are only allowed. Within the first Born approximation the scattering amplitude is written as;

$$
\begin{aligned}
\left|T_{\mathbf{k}, \mathbf{k}^{\prime}}^{+}\right|^{2} & =\left|T_{\mathbf{k}, \mathbf{k}^{\prime}}^{--}\right|^{2}=\frac{2 \pi^{2} J_{0}^{2} S^{2}}{A^{2}}\left|\tilde{T}_{\phi, \phi^{\prime}}\right|^{2}, \\
\left|\tilde{T}_{\phi, \phi^{\prime}}\right|^{2} & =\frac{\xi^{4}}{\left(1+q^{2} \xi^{2}\right)^{3}}\left(1-\cos 2 \theta \cos \phi \cos \phi^{\prime}-\sin \phi \sin \phi^{\prime}\right),
\end{aligned}
$$



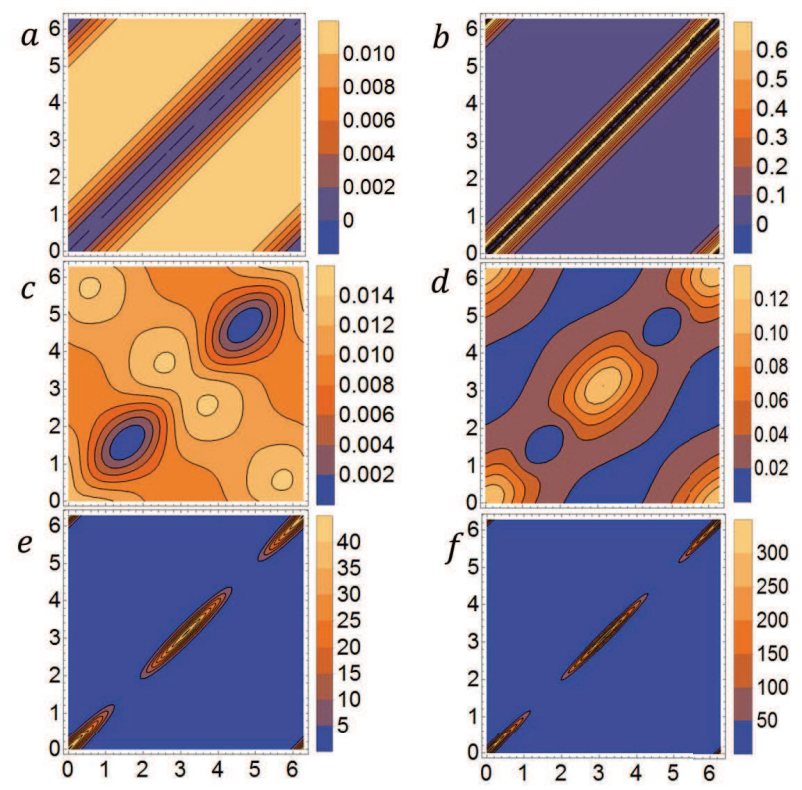

FIG. 2. (Color online) Contour plots of the scattering amplitude, $\left|\tilde{T}_{\phi, \phi^{\prime}}\right|^{2}$ versus $\phi$ (horizontal axes) and $\phi^{\prime}$ (vertical axes), for different values of $k_{\mathrm{F}} \xi$ and $\theta$. In $a$ and $b, k_{\mathrm{F}} \xi$ are, respectively, 0.4 and 3 , and the spins of magnetic clusters are normal to the surface of the TI $(\theta=0)$. Independent to the CMS, the forward scattering amplitudes $\left|\tilde{T}_{\phi, \phi}\right|^{2}$ are always zero, however the backscattering amplitudes $\left|\tilde{T}_{\phi, \pi+\phi}\right|^{2}$ strongly depend on the CMS. In $c, d, f$ and $e$, the values of $k_{\mathrm{F}} \xi$ are, respectively, $0.4,0.7,3$ and 5 , and the tilt angle is $\theta=\pi / 6$. In the presence of clusters with small sizes, the scattering amplitude is zero in the two areas around $\left(\phi, \phi^{\prime}\right)=(\pi / 2, \pi / 2)$ and $(3 \pi / 2,3 \pi / 2)$ (the blue areas), which means that small clusters scatter electrons over a wide area. When clusters become larger the areas with zero scattering amplitude grow rapidly and electrons scatter by clusters in forward directions.

where $q=\left|\mathbf{k}-\mathbf{k}^{\prime}\right|=k \sqrt{2(1-\cos \Delta \phi)}$. At $\theta=0$, the spins of magnetic clusters are normal to the surface of TI and the scattering amplitude depends only on $\Delta \phi$. At $\theta \neq 0$, the situation is however different and the scattering amplitude depends on $\phi$ and $\phi^{\prime}$ (instead of $\Delta \phi$ ), as shown in Fig. 2. In this case the system is anisotropic which is a simultaneous effect of the spin-orbit locking of Dirac electrons and the spin tilting of magnetic clusters. The former is not explicitly seen in the scattering amplitude but the latter is appeared in $\theta$.

Making use of Eqs. (8) and (16), we obtain:

$$
\begin{aligned}
w\left(\phi, \phi^{\prime}\right) & =\frac{1-\cos 2 \theta \cos \phi \cos \phi^{\prime}-\sin \phi \sin \phi^{\prime}}{(1-\Omega \cos \Delta \phi)^{3} W_{0}}, \\
\bar{w}(\phi) & =\frac{2+\Omega^{2}-3 \Omega \cos ^{2} \theta+3 \Omega \sin ^{2} \theta \cos 2 \phi}{\left(1-\Omega^{2}\right)^{5} W_{0}},
\end{aligned}
$$

with

$$
\Omega=\frac{2 k^{2} \xi^{2}}{1+2 k^{2} \xi^{2}}, \quad W_{0}=\frac{\hbar^{2} v_{\mathrm{F}}}{\pi J_{0}^{2} S^{2}} \frac{A}{n_{c}} \frac{\left(1+2 k^{2} \xi^{2}\right)^{3}}{k \xi^{4}},
$$

where $\Omega$ is a dimensionless function, and $W_{0}$ has a di- mension of time.

Substituting the equations (14) and (17) into Eq. (12) results in the following recursive relations for $a_{i}^{c}$ with odd $i$ :

$$
\begin{aligned}
& \left(L_{0}+K_{0}\right) a_{1}^{c}+L_{1} a_{3}^{c}=W_{0}, \\
& L_{m} a_{2 m+1}^{c}+K_{m-1} a_{2 m-1}^{c}+L_{m-1} a_{2 m-3}^{c}=0,
\end{aligned}
$$

where $L_{m}=L_{m}(k, \xi, \theta)$ and $K_{m}=K_{m}(k, \xi, \theta)$ are dimensionless functions (see the Appendix, section A1). By using Kramer's rule and performing some algebraic calculation we obtain:

$$
a_{1}^{c}=\lambda_{0}^{+} W_{0},
$$

with $\lambda_{0}^{+}=\left(L_{0}+K_{0}-\lambda_{1}\right)^{-1}$, where $\lambda_{1}$ is a dimensionless function, depending on $L_{m}$ and $K_{m}$ through the recursive relation:

$$
\lambda_{m}=\frac{\left(L_{m}\right)^{2}}{K_{m}-\lambda_{m+1}} .
$$

Since $\lambda_{0}^{+}$converges by increasing $m$, we can approximate $\lambda_{1}$ by truncating the series at some point (see Appendix, section A 2). Higher-order coefficients $a_{i}^{c}$ with odd $i$ are obtained from Eqs. (19) and (20).

The recursive relations for $a_{i}^{c}$ with even $i$ are also:

$$
\begin{aligned}
& L_{1}^{\prime} a_{2}^{c}+K_{0}^{\prime} a_{0}^{c}=0, \\
& \left(L_{0}^{\prime}+L_{1}^{\prime}\right) a_{0}^{c}+K_{1}^{\prime} a_{2}^{c}+L_{2}^{\prime} a_{4}^{c}=0, \\
& L_{m}^{\prime} a_{2 m}^{c}+K_{m-1}^{\prime} a_{2 m-2}^{c}+L_{m-1}^{\prime} a_{2 m-4}^{c}=0, \quad m \geq 3
\end{aligned}
$$

where the functions $L_{m}^{\prime}=L_{m}^{\prime}(k, \xi, \theta)$ and $K_{m}^{\prime}=$ $K_{m}^{\prime}(k, \xi, \theta)$ are presented in the Appendix, section A 1. The number of Dirac electrons is conserved in scattering processes, so it is necessary the coefficient $a_{0}^{c}$ to be zero which results in $a_{m}^{c}=0$ with even $m$.

The recursive relations for odd and even $a_{i}^{s}$ are also given by:

$$
\begin{aligned}
& \left(K_{0}-L_{0}\right) a_{1}^{s}+L_{1} a_{3}^{s}=0, \\
& L_{m} a_{2 m+1}^{s}+K_{m-1} a_{2 m-1}^{s}+L_{m-1} a_{2 m-3}^{s}=0, \quad m \geq 2 \\
& K_{1}^{\prime} a_{2}^{s}+L_{2}^{\prime} a_{4}^{s}=0, \\
& L_{m}^{\prime} a_{2 m}^{s}+K_{m-1}^{\prime} a_{2 m-2}^{s}+L_{m-1}^{\prime} a_{2 m-4}^{s}=0 . \quad m \geq 3
\end{aligned}
$$

One can show that all the coefficients $a_{i}^{s}$ are zero (see the appendix). Finally, the function $a(\phi)$ is obtained in terms of $a_{i}^{c}=a_{i}^{c}(k, \xi, \theta)$ with odd $i$ as:

$$
a(\phi)=\sum_{n=0}^{\infty} a_{2 n+1}^{c} \cos (2 n+1) \phi .
$$

By analogy with the above derivation, we have also obtained all the coefficients in $b(\phi)$ and found that except of $b_{i}^{s}$ with odd $i$, the others are zero (see the Appendix). All these nonzero coefficients are given in terms of $b_{1}^{s}=\lambda_{0}^{-} W_{0}$ with $\lambda_{0}^{-}=\left(K_{0}-L_{0}-\lambda_{1}\right)^{-1}$. The function $b(\phi)$ is thus written as:

$$
b(\phi)=\sum_{n=0}^{\infty} b_{2 n+1}^{s} \sin (2 n+1) \phi .
$$


Substituting the obtained functions $a(\phi)$ and $b(\phi)$ into Eq. (9), one can obtain the non-equilibrium distribution function $f$.

\section{SURFACE CONDUCTIVITIES}

In the presence of an applied electric field along $j$ direction $\left(E_{j}\right)$, the surface conductivity of the TI along $i$-direction can be obtained from the following relation:

$$
\sigma_{i j}=\frac{e}{E_{j}} \sum_{n} \int \frac{d^{2} k}{(2 \pi)^{2}} \mathbf{v}_{\mathbf{k}}^{n i} f(\mathbf{k}, \mathbf{E}),
$$

where $n$ is the band index, $i$ and $j$ are $x$ and $y$, and $\mathbf{v}_{\mathbf{k}}^{n i}$ is the group velocity of the $n$-th band. By considering intra-band scattering, since $\mathbf{v}_{\mathbf{k}}=v_{\mathbf{k}}(\cos \phi$, sin $\phi)$, the conductivities in $x$ and $y$ directions reduce to

$$
\begin{aligned}
\sigma_{x x} & =\frac{e^{2}}{4 \pi} \int v_{\mathbf{k}}^{2}\left(\frac{\partial f^{0}}{\partial \varepsilon}\right) a_{1}^{c}\left(k, \xi, \theta, n_{c}\right) k d k, \\
\sigma_{y y} & =\frac{e^{2}}{4 \pi} \int v_{\mathbf{k}}^{2}\left(\frac{\partial f^{0}}{\partial \varepsilon}\right) b_{1}^{s}\left(k, \xi, \theta, n_{c}\right) k d k .
\end{aligned}
$$

The above relations show that the conductivities $\sigma_{x x}$ and $\sigma_{y y}$ depend only on the coefficients $a_{1}^{c}$ and $b_{1}^{s}$, respectively. This means that these coefficients are acting as momentum relaxation times of Dirac electrons and play central role on the transport properties of the system. They are actually the effective relaxation times along $x$ and $y$ directions which depend on the CMS $\xi$, the CN $n_{c}$, the tilt angle $\theta$ and the magnitude of the incident electrons' momentum $k$. In the rest of the paper we use the following nomenclature $\tau^{x}=a_{1}^{c}$ and $\tau^{y}=b_{1}^{s}$.

The surface conductivities $\sigma_{x x}$ and $\sigma_{y y}$ depend on temperature via the CMS, the $\mathrm{CN}$ and the derivative of the Fermi-Dirac distribution function $\partial f^{0} / \partial \varepsilon$. Using Monte Carlo simulation one can show that the temperature dependence of $\xi$ and $n_{c}$ is limited to a temperature window around a critical temperature $T_{c}$ which is two orders of magnitude smaller than the Fermi temperature $T_{\mathrm{F}}$. For example, for the three dimensional $\mathrm{TI} \mathrm{Bi}_{2} \mathrm{Se}_{3}$ doped with Fe atoms, the surface is ferromagnetically ordered up to $T_{c} \simeq 100 \mathrm{~K}$ which is small in comparison with the Fermi temperature $T_{\mathrm{F}} \simeq 3500 \mathrm{~K}^{36}$. The temperatures where $\xi$ and $n_{c}$ vary, are much smaller than the Fermi temperature and we can approximate the derivative of the FermiDirac distribution function, $\partial f^{0} / \partial \varepsilon$, with the Dirac delta function $\delta\left(\varepsilon-\varepsilon_{\mathrm{F}}\right)$. With the above considerations the surface conductivities read:

$$
\begin{aligned}
& \sigma_{x x}=\frac{e^{2}}{2 h} k_{\mathrm{F}} v_{\mathrm{F}} \tau_{k_{\mathrm{F}}}^{x}=\sigma_{0} \frac{\left(1+2\left(k_{\mathrm{F}} \xi\right)^{2}\right)^{3}}{\left(k_{\mathrm{F}} \xi\right)^{4}} \lambda_{0}^{+}, \\
& \sigma_{y y}=\frac{e^{2}}{2 h} k_{\mathrm{F}} v_{\mathrm{F}} \tau_{k_{\mathrm{F}}}^{y}=\sigma_{0} \frac{\left(1+2\left(k_{\mathrm{F}} \xi\right)^{2}\right)^{3}}{\left(k_{\mathrm{F}} \xi\right)^{4}} \lambda_{0}^{-},
\end{aligned}
$$

where $\sigma_{0}=\frac{\hbar^{2} v_{\mathrm{F}}^{2} k_{\mathrm{F}}^{4} A}{2 \pi n_{c} J_{0}^{2} S^{2}} \frac{e^{2}}{h}$ is a function of $n_{c}$ with a dimension of conductivity. The conductivity $\sigma_{0}$ is typically at order of $\left(10^{15}-10^{17}\right) \tilde{n}_{c}^{-1} e^{2} / h$, where $\tilde{n}_{c}$ is clusters density. At temperatures where $\mathrm{CN}$ is very large, $\tilde{n}_{c}$ is at order of $10^{13} m^{-2}$ and $\sigma_{0} \sim\left(10^{2}-10^{4}\right) \frac{e^{2}}{h}$. As it is seen from Eqs. (29) and (30), surface conductivities depend on the dimensionless parameter $k_{\mathrm{F}} \xi$. This implies that the ratio of the CMS $\xi$ to the Fermi wavelength of Dirac electrons $\lambda_{\mathrm{F}}=2 \pi / k_{\mathrm{F}}$, determines the measure of surface conductivities. As we will see below, when $\lambda_{\mathrm{F}}$ is comparable with $\xi$, the effective relaxation times and consequently the surface conductivities change behavior.

\section{A. Isotropic case $(\theta=0)$}

When the spins of magnetic clusters are normal to the surface of the TI, the scattering amplitude depends only on $\Delta \phi$ and the system is isotropic. In this case the functions $L_{0}$ and $\lambda_{1}$ are zero and the effective relaxation times $\tau^{x}$ and $\tau^{y}$, correspond exactly to the real relaxation time $\tau=W_{0} / K_{0}$, which could also be resulted from the standard formula. In this case the conductivity is obtained as:

$$
\sigma_{x x}=\sigma_{y y}=\sigma=\frac{\sigma_{0}}{3 \pi} \frac{\left(1+4 k_{\mathrm{F}}^{2} \xi^{2}\right)^{\frac{5}{2}}}{k_{\mathrm{F}}^{4} \xi^{4}}
$$

While $\sigma$ has a simple dependence on $n_{c}$, its variation with $\xi$ is more complicated, as shown in Fig. 3. Note that $\sigma$ decreases initially with increasing $k_{\mathrm{F}} \xi$, becomes minimum at a certain value of $k_{\mathrm{F}} \xi$ and then increases at larger values of $k_{\mathrm{F}} \xi$. Such a dependence arises mainly

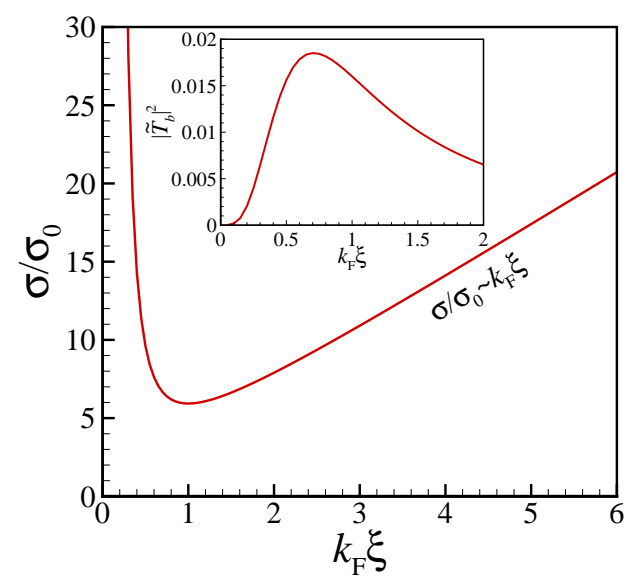

FIG. 3. (color online) The surface conductivity of the TI versus $k_{\mathrm{F}} \xi$ for $\theta=0$. Inset: backscattering amplitude at $\theta=0$.

from the dependence of the $T$-matrix elements, as will be explained below. The $T$-matrix is a multiplication of two parts: one is

$$
\tilde{T}_{\theta}=\cos \theta\left[e^{i \Delta \phi}-1\right]+\sin \theta\left[e^{i \phi}-e^{-i \phi^{\prime}}\right],
$$


which depends on the direction of the clusters' spin $S$ ( $\theta$-dependent part) and the other is

$$
\tilde{T}_{\xi}=\int d \mathbf{r} e^{-i \mathbf{k} \cdot \mathbf{r}} e^{-r / \xi} e^{i \mathbf{k}^{\prime} \cdot \mathbf{r}},
$$

depending on the CMS ( $\xi$-dependent part). For large enough values of $\xi$ (i.e. for $\xi \gg \lambda_{\mathrm{F}}$ ) the scattering potential goes to unity and $\tilde{T}_{\xi}$ is proportional to the Dirac delta function $\delta\left(\mathbf{k}-\mathbf{k}^{\prime}\right)$, meaning that in the limit of infinite $k_{\mathrm{F}} \xi$, only forward scatterings contribute to $\tilde{T}_{\xi}$. On the other hand, from the $\theta$-dependent part we see however that at $\theta=0, \tilde{T}_{\theta}$ is $\left(e^{i \Delta \phi}-1\right)$, which is zero for $\Delta \phi=0$ and hence forward scattering has no contribution to $\tilde{T}_{\theta}$. The $T$-matrix thus goes to zero for $k_{\mathrm{F}} \xi \gg 1$, which results in an infinite surface conductivity. When CMS becomes smaller, the conductivity decreases and becomes minimum exactly at $k_{\mathrm{F}} \xi=1$. This dependence is mainly ascribed to the behavior of the backscattering amplitude. Actually, when clusters shrink, backward scattering is more likely to be occurred in the system (see Fig. 2-a and $b$ ). Backscattering amplitude increases by decreasing $\xi$ with proportion to $\left|\tilde{T}_{b}\right|^{2}=2\left(k_{\mathrm{F}} \xi\right)^{4} /\left[1+4\left(k_{\mathrm{F}} \xi\right)^{2}\right]^{3}$ (see Fig. 3, inset plot), causing reduction of conductivity. For small values of $\xi$ (i.e. for $\left.\xi \ll \lambda_{\mathrm{F}}\right) \exp (-r / \xi)$ is almost zero and Dirac electrons do not feel the scattering potential. In this limit, the same as large- $\xi$ limit, the surface conductivity is infinite. When clusters become larger, the scattering potential increases and consequently the conductivity decreases first with proportion to $\left(k_{\mathrm{F}} \xi\right)^{-4}$, passes through the minimum at $k_{\mathrm{F}} \xi=1$, and then increases with proportion to $k_{\mathrm{F}} \xi$. This indicates that at $\theta=0$ electrons are most efficiently scattered at particular value of $k_{\mathrm{F}} \xi(=1)$.

The reason behind such a behavior of the surface conductivity can be also explained as follows. The surface electron velocity operator is directly proportional to the spin operator $\mathbf{s}=\frac{\hbar}{2} \boldsymbol{\sigma}$ as $\mathbf{v}=\frac{2}{\hbar} v_{\mathrm{F}}(\hat{z} \times \mathbf{s})$. While the TI has a vanishing equilibrium spin expectation, a finite current density $\left(\mathbf{J}=e n\langle\mathbf{v}\rangle_{n e q}\right)$ at the surface of the TI yields a spin density $\langle\mathbf{s}\rangle_{n e q}=\frac{\hbar}{2 e v_{\mathrm{F}}}\left(J_{y} \hat{x}-J_{x} \hat{y}\right)$, where $e$ and $n$ are respectively the electron's charge and density. According to the relation of the spin density with current density, the surface conductivities are proportional to the spin expectation as $\sigma_{x x} \propto\left\langle s_{y}\right\rangle_{n e q}$ and $\sigma_{y y} \propto\left\langle s_{x}\right\rangle_{n e q}$. In the two extreme limits of $\xi \rightarrow 0$ and $\xi \rightarrow \infty$, when the CMS is respectively very smaller and very larger than the Fermi wavelength, actually no-scattering happens in the system and the surface conductivity is very large. The large conductivity for large values of $\xi$ could be explained by the magnetization of the clusters. Actually, when magnetic clusters are very large the magnitude of their spins is also very large and therefore because of the exchange interaction the spin density goes to its maximum value which results in a large surface conductivity. When the CMS increases from zero or in the other side decreases from a large value, the conductivity decreases and the two branches cross at $k_{\mathrm{F}} \xi=1$. At this point since $2 \pi \xi=\lambda_{\mathrm{F}}$, the Fermi wavelength is equal to the circumference of the cluster and an electron standing wave form within the cluster and therefore a minimum appears on the surface conductivity.

\section{B. Anisotropic case $(\theta \neq 0)$}

Now suppose that the spins of magnetic clusters are tilted away from the $z$-axis along the $\theta \neq 0$ direction. In this case the system is highly anisotropic and the surface conductivities dependence to clusters' parameters: $\xi$ and $\theta$ is nontrivial, as shown in Figs. 4 and 5. The same
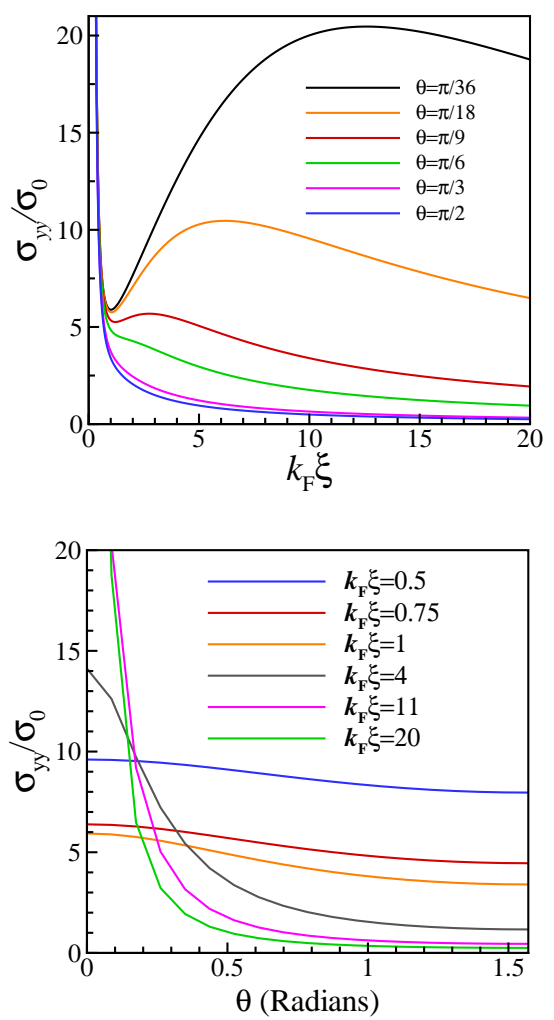

FIG. 4. (Color online) The surface conductivity of the TI along $y$ direction (the direction parallel with the surface of the clusters' spin). Top: versus $k_{\mathrm{F}} \xi$, for different values of $\theta$, and bottom: versus $\theta$, for different CMS. For each value of $k_{\mathrm{F}} \xi, \sigma_{y y}$ decreases monotonically by increasing $\theta$ and becomes minimum at $\theta=\pi / 2$.

as the isotropic case, independent of the values of the tilt angle $\theta$, the conductivity $\sigma_{x x}$ decreases initially by increasing $\xi$, becomes minimum at $k_{\mathrm{F}} \xi=1$ and then increases towards a finite value at $k_{\mathrm{F}} \xi \gg 1$. The variation of $\sigma_{y y}$ by $\xi$ and $\theta$ is however different. For $\theta \leq \pi / 4$, it decreases sharply by increasing $\xi$, becomes minimum at $k_{\mathrm{F}} \xi=1$ and then passes through a broad maximum and finally decreases towards zero at $k_{\mathrm{F}} \xi \gg 1$. For $\theta>\pi / 4$, it decreases sharply by $\xi$, changes dependence at $k_{\mathrm{F}} \xi=1$ and goes to zero smoothly at $k_{\mathrm{F}} \xi \gg 1$. 

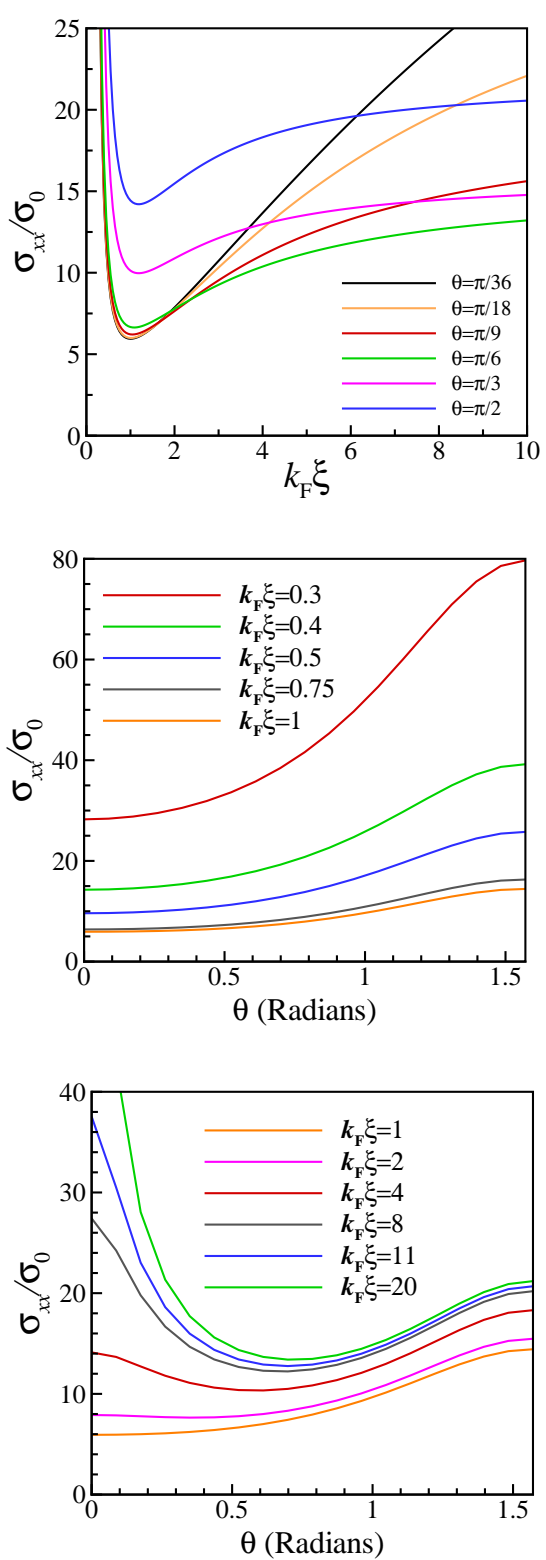

FIG. 5. (Color online) The surface conductivity of the TI along $x$ direction (the direction normal to the surface of the clusters' spin). Top: versus $k_{\mathrm{F}} \xi$, for different values of $\theta$, middle and bottom: versus $\theta$, for different CMS. For small $k_{\mathrm{F}} \xi, \sigma_{x x}$ increases monotonically by increasing $\theta$ and becomes maximum at $\theta=\pi / 2$. For larger $k_{\mathrm{F}} \xi$, it has non-monotonic behavior.

The non-monotonic behaviors of $\sigma_{y y}$ (the oscillatorylike decreasing for small $\theta$, and the non-oscillatory decreasing for larger $\theta$ ) are mainly ascribed to the variation of forward and backward scattering amplitudes. For $\theta \neq 0$, the forward and backward scattering amplitudes are simplified to

$$
\begin{aligned}
\left|\tilde{T}_{f}\right|^{2} & =\left(k_{\mathrm{F}} \xi\right)^{4}\left(1-\cos 2 \theta \cos ^{2} \phi-\sin ^{2} \phi\right), \\
\left|\tilde{T}_{b}\right|^{2} & =\frac{\left(k_{\mathrm{F}} \xi\right)^{4}}{\left(1+4\left(k_{\mathrm{F}} \xi\right)^{2}\right)^{3}}\left(1+\cos 2 \theta \cos ^{2} \phi+\sin ^{2} \phi\right)
\end{aligned}
$$

When the external electric field is applied along the $y$

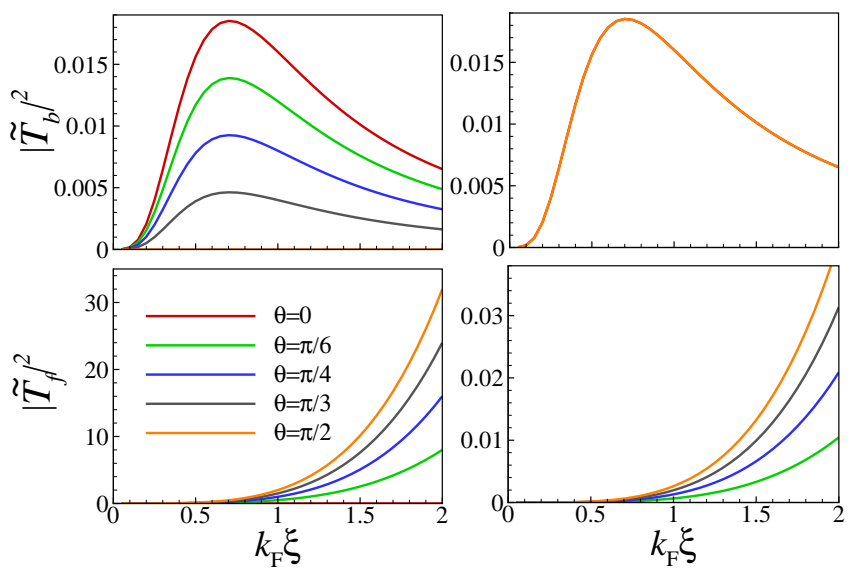

FIG. 6. (Color online) The backscattering (top) and the forward scattering (bottom) amplitudes versus $k_{\mathrm{F}} \xi$, for different values of $\theta$. Left column: for $\phi \simeq 0$, and right column: for $\phi \simeq \pi / 2$.

direction, the main contribution to the conductivity $\sigma_{y y}$ comes from electrons whose momentums are along $y$ direction $(\phi=\pi / 2)$ and hence their spins are aligned in $x$ direction. In this case, the backscattering amplitudes are identically the same for all values of $\theta$, as shown in Fig. 6 , the top-right panel. It is first zero at $\xi=0$, increases by increasing $\xi$, becomes maximum around $k_{\mathrm{F}} \xi=1$ and then decreases gradually towards zero by increasing $\xi$. This is in part due the fact that when the spin of electrons lies on $x$ direction, it is normal to the $y z$ plane and the magnetic torque exerted on the electrons $(|\mathbf{S} \times \boldsymbol{\sigma}|)$ does not vary by increasing the tilt angle $\theta$ (see Fig. 7).

The forward scattering amplitudes depend however on $\theta$. For all values of $k_{\mathrm{F}} \xi$, they are zero at $\theta=0$, increase monotonically by increasing $\theta$ and become maximum at $\theta=\pi / 2$ (see Fig. 6). For small values of $\xi$, backscattered electrons have the main contribution to determine the behavior of the conductivity $\sigma_{y y}$, whereas for larger $\xi$, forward scattered electrons play the main role. Competition between forward and backward scattering amplitudes specify the dependence of the surface conductivity $\sigma_{y y}$ to $\xi$. In order to compare these two amplitudes and to find out the origin of the minimum/maximum appeared on $\sigma_{y y}$, we have also plotted in Fig. 8, the total amplitude $\left|\tilde{T}_{f}\right|^{2}+\left|\tilde{T}_{b}\right|^{2}$ versus $k_{\mathrm{F}} \xi$, for different tilt angles $\theta$. As shown in Fig. 8, the maximum at $k_{\mathrm{F}} \xi=1$ and the minimum emerged at $k_{\mathrm{F}} \xi>1$, are respectively the reasons of the minimum and the maximum of $\sigma_{y y}$ for $\theta>\pi / 4$ and also the reasons of changing the dependence to $k_{\mathrm{F}} \xi$ at $k_{\mathrm{F}} \xi=1$, for $\theta \leq \pi / 4$. 

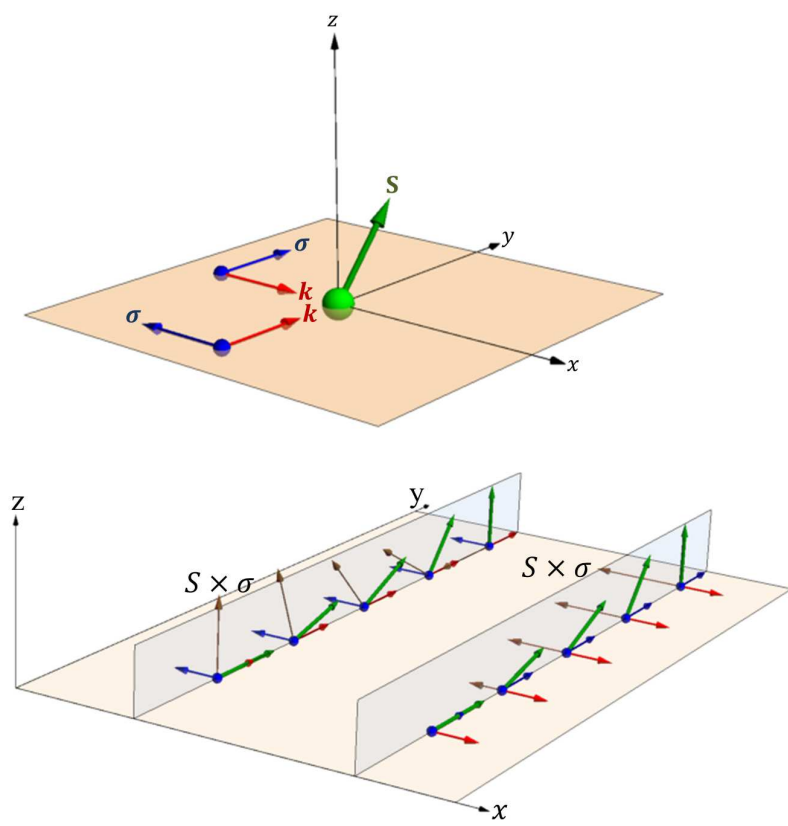

FIG. 7. (Color online) The schematic illustration of the torque exerted on the spins of Dirac electrons by a magnetic spin. The red and blue arrows are, respectively, the momentum and spin of Dirac electrons, the green arrows are spins of magnetic clusters and the browns are the torque exerted on electrons. When electrons move along $y$ direction, their spins are normal to the spin of magnetic clusters and the torque magnitude $(|\mathbf{S} \times \boldsymbol{\sigma}|)$ does not change. However, when the electrons move along $y$ direction, their spins are in $y$ direction and the torque depends on the tilt angle $\theta$, decreasing by $\theta$ and becomes zero at $\theta=\pi / 2$.

When the electric field is applied along the $x$ direction, the main contribution to the transport comes from electrons whose momentums are along $x$ axis. By increasing $\theta$ from 0 to $\pi / 2$, the magnetic torque exerted on Dirac electrons by magnetic clusters decreases from its maximum value towards zero (see Fig. 7) and consequently the backscattering amplitudes decrease (see Fig. 6, topleft panel), causing the increase of the minimum in $\sigma_{x x}$. In contrast with $\sigma_{y y}$, the conductivity $\sigma_{x x}$ doesn't have a monotonic behavior with respect to $\theta$. For $k_{\mathrm{F}} \xi \leq 1$, $\sigma_{x x}$ increases by increasing $\theta$ and becomes maximum at $\theta=\pi / 2$ where all magnetic spins lie on the surface of the TI. This behavior is however not seen for larger values of $k_{\mathrm{F}} \xi$. For $k_{\mathrm{F}} \xi>1, \sigma_{x x}$ decreases first by increasing $\theta$, passes through a minimum, roughly around $\theta=\pi / 4$, and becomes maximum at $\theta=\pi / 2$. The behavior of the conductivity $\sigma_{y y}$ is mainly ascribed to the behavior of the forward and backward scattering amplitudes, however for the conductivity $\sigma_{x x}$, there is no clear-cut reason and aside from these amplitudes others have also considerable contributions in $\sigma_{x x}$.

It should be noted that, while the spin of magnetic clusters exert a torque on the spin of itinerant electrons, we expect that the itinerant spins also conversely exert a

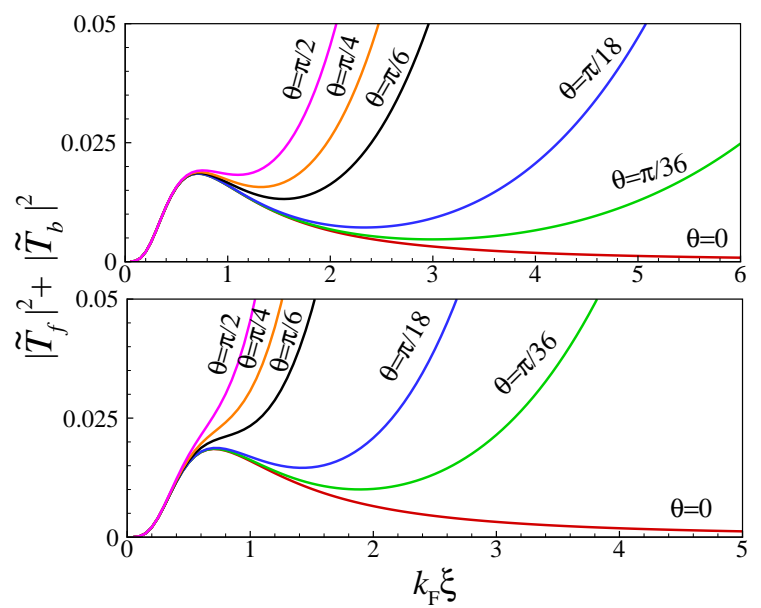

FIG. 8. (Color online) The total forward and backward scattering amplitudes versus $k_{\mathrm{F}} \xi$, for different values of $\theta$, when external electric field is applied along $y$ direction. In this case most of surface electrons move along $y$ direction and the incident angle is a cute angle around $\phi=\pi / 2$. Top: $\phi=92^{\circ}$, and bottom: $\phi=97^{\circ}$.

torque on the magnetic spins. However, in our system the spin of magnetic clusters is approximated as a classical spin vector which has no variations in space and time and its dynamics is much slower than that of itinerant spins. So, the spin torque driven by the exchange interaction only slightly modifies the damping parameter and can not be identified as a leading mechanism for magnetic spin damping. ${ }^{37}$

Now we compare our results with the recent theoretical studies of the scattering of the Dirac electrons with magnetic skyrmions. ${ }^{38,39}$ In Ref. [38], Araki and Nomura used a hard-wall skyrmion approximation in which the magnetization unit vector at the location $\mathbf{r}=(\rho, \phi)$ is approximated by $\left(-\sqrt{1-n_{z}(\rho)} \sin \phi, \sqrt{1-n_{z}(\rho)} \cos \phi, n_{z}(\rho)\right)$ where $n_{z}(\rho)=\operatorname{sgn}\left(\rho-R_{s}\right)$, with $R_{s}$ the radius of the skyrmion, and fully solve the scattering problem by a hard-wall magnetic skyrmion, and estimate its effect on the anomalous Hall conductivity using the Boltzmann transport theory. According to this approximation the Dirac electrons move on the surface of the TI at the presence of an out-of-plane external magnetization along positive $\hat{z}$-direction and scattered off a curved hard-wall. The transmitted electrons to the inside of the skyrmion feel an out-of-plane magnetization along negative $\hat{z}$-direction. Therefore the time reversal symmetry of the system is broken and the band structure is gapped, so the topological Hall effect is seen owing to the skew scattering ${ }^{40}$ of the massive Dirac electron from the hard-wall potential. Moreover,the scattering of Dirac electrons with hard-wall skyrmions is isotropic, and therefore the surface conductivities satisfy the relations $\sigma_{x x}=\sigma_{y y}$ and $\sigma_{x y}=\sigma_{y x}$. In our work magnetic clusters with tilted spins cause the system to be anisotropic and since Dirac electrons are 
massless, the anomalous Hall effect is not seen.

In Ref. [39], it has been studied numerically the longitudinal conductance of a 3D TI/ferromagnet (FM) bilayer where the FM supports different types of individual skyrmions. Regarding the skyrmion as a fixed texture with its center coinciding with the center of the FM and its spin direction given by

$$
\mathbf{m}(\mathbf{r})=(\sin \Theta(r) \cos \Phi(\phi), \sin \Theta(r) \sin \Phi(\phi), \cos \Theta(r)),
$$

where $\Theta(r) \propto e^{-r / R_{s}}$, with $R_{s}$ being the size of skyrmion, it has been shown that in comparison with a trivial ferromagnetic texture where the magnetization is uniform and in the $\hat{z}$ direction, the skyrmion textures can lead to a change of the longitudinal resistance of the order of $k \Omega$. Moreover, the parameter $\Delta G$ defined as the difference of the conductance of the TI in the presence of a nonuniform skyrmion texture and a uniform trivial texture, as a function of the input voltage $V_{i n}$ depends on the FM dimensions and the skyrmion type and size. They showed that for $V_{i n} \leq 60 \mathrm{meV}, \Delta G$ linearly increases by $\frac{\pi R_{s}^{2}}{L W}$, where $L$ and $W$ are respectively the length and width of the FM layer. Similarly, in our system, the surface conductivity is a nonlinear function of the CMS, however its dependence to the CMS is more complex, originating form the difference in the scattering potentials.

\section{Anisotropic magnetoresistance (AMR)}

As a measure of anisotropy, we have also plotted in Fig. 9 the AMR of the TI versus $k_{\mathrm{F}} \xi$ for different values of the tilt angle $\theta$. Typically, AMR is a criterion for the amount of anisotropy and is defined $\mathrm{as}^{35}$

$$
A M R=\frac{\sigma_{x x}-\sigma_{y y}}{\sigma_{x x}+\sigma_{y y}} .
$$

Using Eqs. (29) and (30), the AMR is obtained as:

$$
A M R=\frac{L_{0}}{\lambda_{1}-K_{0}} .
$$

The AMR increases gradually by increasing $k_{\mathrm{F}} \xi$ and approaches to unity for large values of $k_{\mathrm{F}} \xi$, as shown in Fig. 9. At large values of $k_{\mathrm{F}} \xi$, independent of the tilt angle $\theta$, the conductivity along $y$ direction is almost zero and hence $A M R=1$. By increasing the tilt angle $\theta$, the AMR also increases, indicating that the system is more anisotropic when the clusters' spins lie on the surface of the TI. In this case, the spins of clusters are parallel with the spin of Dirac electrons moving along $x$-axis and is normal to the spin of electrons moving along $y$-axis. This will make in turn the difference in the conductivity in two directions and results in an enhancement of the AMR by increasing $\theta$.

In Fig. 10, we have also plotted the AMR of the TI versus the tilt angle $\theta$ for different values of the CMS. For comparison, we have also plotted the AMR of the magnetically doped TI where the scattering potential is the

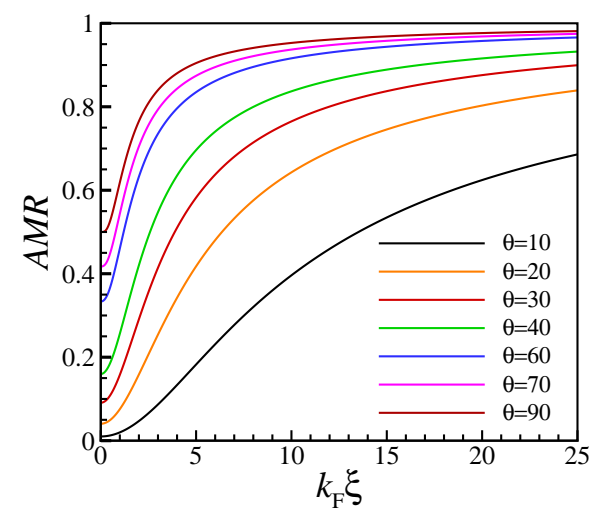

FIG. 9. (Color online) Anisotropic magneto-resistance versus $k_{\mathrm{F}} \xi$, for different values of the tilt angle $\theta$.

short-range Dirac delta function ${ }^{21}$, i.e., the interaction of the Dirac electron located at $\mathbf{r}$ with the impurity at $\mathbf{R}$ is

$$
V=J_{0} \boldsymbol{\sigma}(\mathbf{r}) \cdot \mathbf{S}(\mathbf{R}) \delta(\mathbf{r}-\mathbf{R}),
$$

where $J_{0}$ is coupling constant. In sharp contrast with our system, in this case the surface conductivity $\sigma_{y y}$ is $\theta$-independent, and consequently the AMR is given by $A M R=\sin ^{2} \theta /\left(2+\cos ^{2} \theta\right)$, where $\theta$ is the tilt angle of the magnetic impurities with the $z$-axis normal to the surface of the TI. ${ }^{21}$ But, as shown in Fig. 10, taking into account the effects of impurity-impurity interaction by means of magnetic clusters, modifies this result significantly and the conventional angular dependence of the AMR does not work. In the presence of magnetic clusters, for all

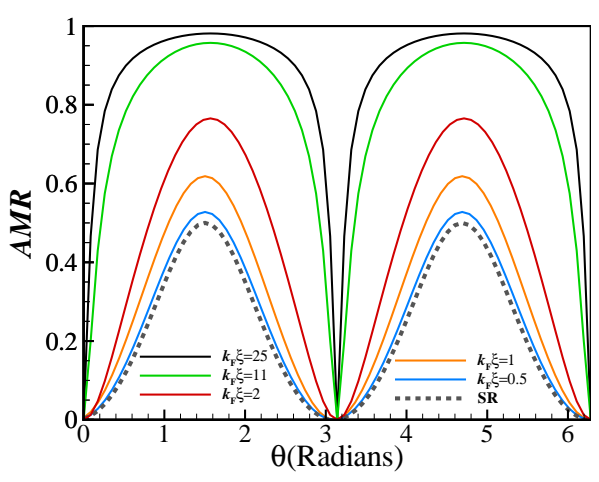

FIG. 10. The angular dependence of the AMR, for different values of CMS. The bold dotted line is the AMR of the TI doped with magnetic impurities, where the scattering potential is the short-range Dirac delta function.

values of $\theta$, the $\mathrm{AMR}$ increases by increasing the CMS and the system experiences a giant AMR $\sim 1$ at $\theta=\pi / 2$, where all magnetic spins lie on the surface of the TI.

Our results are consistent with the recent experimental work on the AMR of the Cr-doped (Bi, Sb) ${ }_{2} \mathrm{Te}_{3}$ TI. ${ }^{26} \mathrm{In}$ Ref. [26], it has been shown that for a $1 \mathrm{~T}$ magnetic field 
the AMR, defined as $\left(\rho_{x x, \max }-\rho_{x x, \min }\right) / \rho_{x x, \min }$, takes a maximum value $\sim 140 \%$, and its angular dependence is completely inconsistent with the well-known $\cos ^{2} \theta$ angular dependence seen in conventional ferromagnets, where $\theta$ is the tilt angle of the magnetic field with $z$-axis. The consistency of our results with experiment confirms that using the concept of magnetic clusters in the TI doped with interacting magnetic impurities, properly gives the transport properties of real magnetic TI.

\section{NONMAGNETIC IMPURITY}

In previous sections, we studied the surface conductivities of the TI doped with magnetic impurities both in the isotropic $(\theta=0)$ and anisotropic $(\theta \neq 0)$ cases, by defining the scattering potential as in Eq. (4), composed of two parts: an spin-dependent part $(\boldsymbol{\sigma} \cdot \mathbf{S})$ and a $\xi$-dependent part $(\exp (-|\mathbf{r}-\mathbf{R}| / \xi))$. We demonstrated that the non-monotonic behavior of the surface conductivities versus $\xi$ is a simultaneous effect of the spin-orbit locking of Dirac electrons, and the spin direction and the CMS. In order to see the effects of the $\xi$-dependent part on the behavior of the surface conductivity separately, in this section we investigate the transport properties of a 3D TI, doped with nonmagnetic impurities.

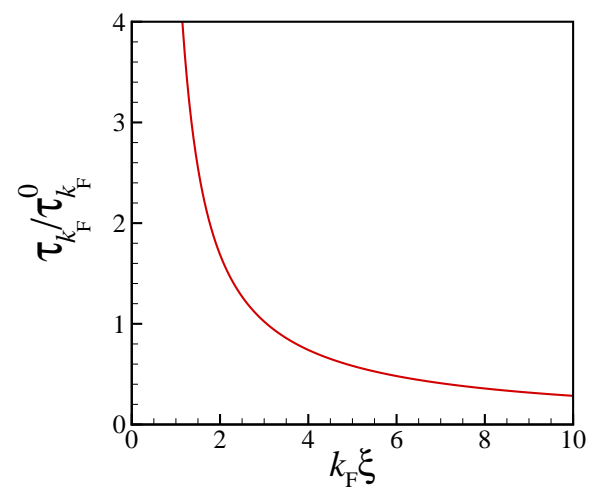

FIG. 11. (Color online) The relaxation time of the TI doped with nonmagnetic impurities versus $k_{\mathrm{F}} \xi$. By increasing $\xi$, the relaxation time decreases monotonically.

Let us suppose that the surface of the TI is doped with non-magnetic impurities and the scattering of an electron located at $\mathbf{r}$ with the impurity centered at $\mathbf{R}$ is given by:

$$
V=V_{0} \exp (-|\mathbf{r}-\mathbf{R}| / \xi),
$$

where $V_{0}$ and $\xi$ are respectively the strength and the range of the scattering potential. The scattering amplitude in this system is given by;

$$
\left|T_{\mathbf{k}, \mathbf{k}^{\prime}}^{++}\right|^{2}=\left|T_{\mathbf{k}, \mathbf{k}^{\prime}}^{--}\right|^{2}=\frac{2 \pi^{2} V_{0}^{2}}{A^{2}} \frac{\xi^{4}}{\left(1+q^{2} \xi^{2}\right)^{3}}(1+\cos \Delta \phi),
$$

which depends only on $\Delta \phi$. The scattering is hence isotropic and the relaxation time is given by the standard formula: $\frac{1}{\tau_{k}}=A \int \frac{d^{2} k^{\prime}}{(2 \pi)^{2}} w\left(\mathbf{k}, \mathbf{k}^{\prime}\right)[1-\cos \Delta \phi]$. Using the $T$-matrix elements in Eq. (40), the relaxation time is obtained as:

$$
\frac{1}{\tau_{k}}=\frac{1}{\tau_{k}^{0}} \frac{k^{4} \xi^{4}}{\left(1+2 k^{2} \xi^{2}\right)^{3 / 2}},
$$

where $\tau_{k}^{0}=\frac{\hbar^{2} v_{\mathrm{F}} A k^{3}}{\pi^{2} n_{c} V_{0}^{2}}$ is a parameter with a dimension of time.

We have plotted in Fig. 11, the dimensionless relaxation time $\tau_{k_{\mathrm{F}}} / \tau_{k_{\mathrm{F}}}^{0}$ of the TI doped with nonmagnetic impurities versus $k_{\mathrm{F}} \xi$. As it is shown, it decreases monotonically towards zero by increasing $k_{\mathrm{F}} \xi$. This behavior is in sharp contrast with magnetic TIs. In the TIs doped with magnetic impurities, the exchange interaction of magnetic impurities with Dirac electrons, causes the system to be different and the non-monotonic behaviors of conductivity (see Fig. 3) to be seen.

\section{SUMMARY AND CONCLUSION}

We have studied the transport properties of a three dimensional topological insulator (TI) doped with magnetic impurities by taking the effects of impurityimpurity exchange interactions into account. The interaction between magnetic impurities gives rise to the formation of magnetic clusters with temperature dependent mean sizes and numbers, randomly distributed on the surface of the TI. We have considered that Dirac electrons scatter by magnetic clusters, rather than single impurities, and defined the scattering potential in terms of CMS. Making use of the semiclassical Boltzmann approach and employing a generalized relaxation time approximation, we have obtained the surface conductivity of the TI by solving four sets of recursive relations. We have demonstrated that the system is highly anisotropic and the surface conductivities strongly depend on the mean size and the spin directions of magnetic clusters. We have shown that, in the isotropic TI, the surface conductivity possesses a non-monotonic behavior, it decreases by increasing CMS, passes through a minimum where the CMS is identically the same as Fermi wave length of Dirac electrons, and increases by increasing CMS with proportion to the $k_{\mathrm{F}} \xi$. We have also shown that, when the system is anisotropic, the behavior of surface conductivities with respect to the CMS and to the clusters' spin directions is nontrivial. Actually, independent of the values of the tilt angle of cluster spins, the conductivity along the direction normal to the surface of clusters' spins decreases by increasing the CMS, becomes minimum at $k_{\mathrm{F}} \xi=1$ and then increases and saturates for larger CMS. The behavior of the conductivity along the direction parallel to the surface of the clusters' spins is however different. We have shown that for tilt angles roughly smaller than $\pi / 4$ it goes oscillatory 
to zero by increasing CMS, and for larger tilt angles it decreases sharply by increasing CMS, changes behavior around $k_{\mathrm{F}} \xi=1$ and decays smoothly to zero for large CMS.

Furthermore, by comparing our results with the relaxation time and the surface conductivity of a TI doped with long-range non-magnetic impurities, we have demonstrated that the minimum appeared on the surface conductivities of the magnetic TI is essentially arises from the spin-dependent part of the potential.

We have also investigated the conductivity of twodimensional semiconductors in the presence of both Rashba and Dresselhous spin-orbit couplings. Such kind of minimum is also emerged on the conductivity of these systems. These results will be presented in our future works.

Since CMS and CN depend on temperature, the conductivity is also temperature dependent. In order to investigate the behavior of the anisotropic magnetoresistance of the magnetic TI versus temperature, it is required to obtain the temperature dependence of the CMS and CN. Computing these functions by means of a quantum Monte Carlo simulation is also left for future works.

In the case that the exchange interaction $J_{0}$ is comparable with the Fermi energy, the higher order terms in the T-matrix should be taken into account on the transport properties of the system. As it has been discussed in Ref. [40], the side-jump effects which follow from the coordinate shifts during the scattering events, and also the skew scattering which depends on the asymmetric part of the scattering rate and is at order of $(\text { Scat. Potential })^{3}$, have non-zero contributions to the surface conductivities of gapped TI. In the TI with the time reversal symmetry, these effects have vanishing contribution to the surface conductivity and the Hall conductivity is zero. In our system, the unperturbed Hamiltonian is gapless and skew scattering, anomalous velocity and the side-jump effects have vanishing contributions to the surface conductivity. However, in the case of gapped TI, where the time reversal symmetry is broken, the system has a non-zero Hall conductivity. Investigation of the anomalous Hall conductivity for our system in the presence of a bulk magnetization is left for the future study.

\section{ACKNOWLEDGMENTS}

The authors would like to thank G. Baskaran for insightful comments on the manuscript. Useful discussions with S. H. Abedinpour and A. G. Moghaddam are acknowledged.

\section{Appendix A: The solution of the inhomogeneous Fredholm integral equations (12) and (13)}

In this appendix we bring the details of our calculations of the functions $a(\phi)$ and $b(\phi)$, appeared in the nonequilibrium distribution function $f$. For obtaining the coefficients in $a(\phi)$ and $b(\phi)$ we should solve the integral equations (12) and (13). By using Fourier series of $a(\phi)$ and $b(\phi)$ and expand the integral kernels in terms of trigonometric functions, the integral equations reduce to four sets of recursive linear equations for the expansion coefficients of Fourier series. By solving the linear equations, we obtain the functions $a(\phi)$ and $b(\phi)$.

\section{Recursive relations between the coefficients appeared in $a(\phi)$ and $b(\phi)$}

Employing the Fourier series of $a(\phi)$ and $b(\phi)$ (Eqs. (14) and (15)), the integrals in Eqs. (12) and (13) are, respectively, written as

$$
\begin{aligned}
\int w\left(\phi, \phi^{\prime}\right) a\left(\phi^{\prime}\right) d \phi^{\prime} & =\sum_{m=0} \int w\left(\phi, \phi^{\prime}\right)\left(a_{m}^{c} \cos m \phi^{\prime}+a_{m}^{s} \sin m \phi^{\prime}\right) d \phi^{\prime}, \\
\int w\left(\phi, \phi^{\prime}\right) b\left(\phi^{\prime}\right) d \phi^{\prime} & =\sum_{m=0} \int w\left(\phi, \phi^{\prime}\right)\left(b_{m}^{c} \cos m \phi^{\prime}+b_{m}^{s} \sin m \phi^{\prime}\right) d \phi^{\prime}
\end{aligned}
$$

By expanding $w\left(\phi, \phi^{\prime}\right)$ (see Eq. 17) as

$$
w\left(\phi, \phi^{\prime}\right)=\frac{\left(1-\cos 2 \theta \cos \phi \cos \phi^{\prime}-\sin \phi \sin \phi^{\prime}\right)}{W_{0}} \sum_{n=0} \frac{(n+2) !}{2 n !} \Omega^{n} \cos ^{n} \Delta \phi,
$$


the first integral on the right hand side of Eq. (A1) is given by:

$$
\begin{aligned}
& \sum_{m=0} \int w\left(\phi, \phi^{\prime}\right) a_{m}^{c} \cos m \phi^{\prime} d \phi^{\prime} \\
& =\frac{1}{W_{0}} \sum_{m, n=0} \int d \phi^{\prime}\left(1-\cos 2 \theta \cos \phi \cos \phi^{\prime}-\sin \phi \sin \phi^{\prime}\right)\left(\frac{(2 n+2) !}{2(2 n) !} \Omega^{2 n} \cos ^{2 n} \Delta \phi+\frac{(2 n+3) !}{2(2 n+1) !} \Omega^{2 n+1} \cos ^{2 n+1} \Delta \phi\right) \times \\
& \left(a_{2 m}^{c} \cos 2 m \phi^{\prime}+a_{2 m+1}^{c} \cos (2 m+1) \phi^{\prime}\right) \\
& =\frac{1}{W_{0}} \sum_{m, n=0} \int d \phi^{\prime}\left(\frac{(2 n+2) !}{2(2 n) !} \Omega^{2 n} \cos ^{2 n} \Delta \phi \cos 2 m \phi^{\prime} a_{2 m}^{c}+\frac{(2 n+3) !}{2(2 n+1) !} \Omega^{2 n+1} \cos ^{2 n+1} \Delta \phi \cos (2 m+1) \phi^{\prime} a_{2 m+1}^{c}\right. \\
& -\cos 2 \theta \cos \phi\left[\frac{(2 n+2) ! \Omega^{2 n}}{4(2 n) !} \cos ^{2 n} \Delta \phi\left(\cos (2 m+2) \phi^{\prime}+\cos 2 m \phi^{\prime}\right) a_{2 m+1}^{c}\right. \\
& \left.+\frac{(2 n+3) ! \Omega^{2 n+1}}{4(2 n+1) !} \cos ^{2 n+1} \Delta \phi\left(\cos (2 m+1) \phi^{\prime}+\cos (2 m-1) \phi^{\prime}\right) a_{2 m}^{c}\right] \\
& -\sin \phi\left[\frac{(2 n+2) ! \Omega^{2 n}}{4(2 n) !} \cos ^{2 n} \Delta \phi\left(\sin (2 m+1) \phi^{\prime}-\sin 2 m \phi^{\prime}\right) a_{2 m+1}^{c}\right. \\
& \left.\left.+\frac{(2 n+3) ! \Omega^{2 n+1}}{4(2 n+1) !} \cos ^{2 n+1} \Delta \phi\left(\sin (2 m+1) \phi^{\prime}-\sin (2 m-1) \phi^{\prime}\right) a_{2 m}^{c}\right]\right)
\end{aligned}
$$

Using the following expansions,

$$
\begin{aligned}
& \cos ^{2 n} \phi=\frac{1}{2^{2 n}}\left(\begin{array}{c}
2 n \\
n
\end{array}\right)+\frac{1}{2^{2 n-1}}\left[\cos 2 n \phi+\left(\begin{array}{c}
2 n \\
1
\end{array}\right) \cos (2 n-2) \phi+\cdots+\left(\begin{array}{c}
2 n \\
n-1
\end{array}\right) \cos 2 \phi\right], \\
& \cos ^{2 n-1} \phi=\frac{1}{2^{2 n-1}}\left[\cos (2 n-1) \phi+\left(\begin{array}{c}
2 n-1 \\
1
\end{array}\right) \cos (2 n-3) \phi+\cdots+\left(\begin{array}{c}
2 n-1 \\
n-1
\end{array}\right) \cos \phi\right],
\end{aligned}
$$

according to the orthogonality of trigonometric functions we have

$$
\begin{aligned}
& \int_{0}^{2 \pi} \cos ^{2 n} \Delta \phi\left\{\begin{array}{l}
\cos 2 m \phi^{\prime} \\
\sin 2 m \phi^{\prime}
\end{array}\right\} d \phi^{\prime}= \begin{cases}\frac{\pi}{2^{2 n-1}}\left(\begin{array}{c}
2 n \\
n-m
\end{array}\right)\left\{\begin{array}{ll}
\cos 2 m \phi \\
\sin 2 m \phi
\end{array}\right\}, & m \leqslant n \\
0, & m \neq n\end{cases} \\
& \int_{0}^{2 \pi} \cos ^{2 n+1} \Delta \phi \cos (2 m+1) \phi^{\prime} d \phi^{\prime}= \begin{cases}\frac{\pi}{2^{2 n}}\left(\begin{array}{c}
2 n+1 \\
n-m
\end{array}\right)\left\{\begin{array}{ll}
\cos (2 m+1) \phi \\
\sin (2 m+1) \phi
\end{array}\right\}, & m \leqslant n \\
0 . & m \neq n\end{cases}
\end{aligned}
$$

By making use of the relations in (A5), we obtain the integral (A4) as:

$$
\begin{aligned}
& \sum_{m=0} \int w\left(\phi, \phi^{\prime}\right) a_{m}^{c} \cos m \phi^{\prime} d \phi^{\prime} \\
& =\frac{1}{W_{0}} \sum_{m=0}\left(\cos (2 m-2) \phi\left[\frac{(1-\cos 2 \theta)}{4} G(m-1, \Omega)\right] a_{2 m}^{c}+\cos (2 m-1) \phi[F(m, \Omega)(1-\cos 2 \theta)] a_{2 m+1}^{c}\right. \\
& +\cos 2 m \phi\left[4 F(m, \Omega)-\frac{(1+\cos 2 \theta)}{4}(G(m-1, \Omega)+G(m, \Omega))\right] a_{2 m}^{c} \\
& +\cos (2 m+1) \phi[G(m+1, \Omega)-(1+\cos 2 \theta)(F(m, \Omega)+F(m+1, \Omega))] a_{2 m+1}^{c} \\
& \left.+\cos (2 m+2) \phi\left[\frac{(1-\cos 2 \theta)}{4} G(m, \Omega)\right] a_{2 m}^{c}+\cos (2 m+3) \phi[(1-\cos 2 \theta) F(m+1, \Omega)] a_{2 m+1}^{c}\right)
\end{aligned}
$$


where

$$
\begin{aligned}
F(m, \Omega)=\sum_{n=0} \frac{(2 n+2) !}{4(n-m) !(n+m) !}\left(\frac{\Omega}{2}\right)^{2 n} & =\sum_{k=0} \frac{(2 m+2 k+2) !}{4 k !(2 m+k) !}\left(\frac{\Omega}{2}\right)^{2 m+2 k} \\
& =\frac{(1+m)(1+2 m)}{2}\left(\frac{\Omega}{2}\right)^{2 m} F_{1}\left[\frac{3}{2}+m, 2+m, 1+2 m, \Omega^{2}\right], \\
G(m, \Omega)=\sum_{n=0} \frac{(2 n+3) !}{(n-m) !(n+m+1) !}\left(\frac{\Omega}{2}\right)^{2 n+1} & =\sum_{k=0} \frac{(2 m+2 k+3) !}{k !(2 m+k+1) !}\left(\frac{\Omega}{2}\right)^{2 m+2 k+1} \\
& =2 m(1+2 m)\left(\frac{\Omega}{2}\right)^{2 m+1} F_{1}\left[2+m, \frac{5}{2}+m, 2+2 m, \Omega^{2}\right] .
\end{aligned}
$$

Here, ${ }_{2} F_{1}\left[\frac{3}{2}+m, 2+m, 1+2 m, \Omega^{2}\right]$ and ${ }_{2} F_{1}\left[2+m, \frac{5}{2}+m, 2+2 m, \Omega^{2}\right]$ are hypergeometric functions. Since, $0 \leq \Omega \leq 1$, the functions $F(m, \Omega)$ and $G(m, \Omega)$ are simplified as;

$$
\begin{aligned}
& F(m, x)=\frac{\pi\left(2+x^{2}+6 m \sqrt{1-x^{2}}-4 m^{2}\left(1-x^{2}\right)\right)}{4 x^{-2 m}\left(1+\sqrt{1-x^{2}}\right)^{2 m}\left(1-x^{2}\right)^{\frac{5}{2}}}, \\
& G(m-1, x)=\frac{3 \pi-3 \pi \sqrt{1-x^{2}}(1-2 m)+4 \pi m(m-1)\left(1-x^{2}\right)}{x^{1-2 m}\left(1+\sqrt{1-x^{2}}\right)^{2 m-1}\left(1-x^{2}\right)^{\frac{5}{2}}} .
\end{aligned}
$$

The second integral on the right hand side of Eq. (A1) is also obtained as:

$$
\begin{aligned}
& \sum_{m=0} \int w\left(\phi, \phi^{\prime}\right) a_{m}^{s} \sin m \phi^{\prime} d \phi^{\prime} \\
& =\frac{1}{W_{0}} \sum_{m=0}\left(\sin (2 m-2) \phi\left[\frac{(1-\cos 2 \theta)}{4} G(m-1, \Omega)\right] a_{2 m}^{s}+\sin (2 m-1) \phi[F(m, \Omega)(1-\cos 2 \theta)] a_{2 m+1}^{s}\right. \\
& +\sin 2 m \phi\left[4 F(m, \Omega)-\frac{(1+\cos 2 \theta)}{4}(G(m-1, \Omega)+G(m, \Omega))\right] a_{2 m}^{s} \\
& +\sin (2 m+1) \phi[G(m+1, \Omega)-(1+\cos 2 \theta)(F(m, \Omega)+F(m+1, \Omega))] a_{2 m+1}^{s} \\
& \left.+\sin (2 m+2) \phi\left[\frac{(1-\cos 2 \theta)}{4} G(m, \Omega)\right] a_{2 m}^{s}+\sin (2 m+3) \phi[(1-\cos 2 \theta) F(m+1, \Omega)] a_{2 m+1}^{s}\right) .
\end{aligned}
$$

Substituting (A6) and (A9) into Eq. (12), and equalizing the coefficients of the trigonometric functions with the same arguments, we reach the recursive relations in Eqs. (19), (22) and (23) which are respectively expressed in the following matrix forms:

$$
\begin{gathered}
{\left[\begin{array}{ccccccc}
L_{0}+K_{0} & L_{1} & 0 & 0 & 0 & \ldots & \\
L_{1} & K_{1} & L_{2} & 0 & 0 & 0 & \ldots \\
0 & L_{2} & K_{2} & L_{3} & 0 & 0 & \ldots \\
0 & 0 & L_{3} & K_{3} & L_{4} & 0 & \ldots \\
0 & 0 & 0 & L_{4} & K_{4} & L_{5} & \ldots \\
\vdots & \vdots & \vdots & \vdots & \vdots & \ddots &
\end{array}\right]\left[\begin{array}{c}
a_{1}^{c} \\
a_{3}^{c} \\
a_{5}^{c} \\
a_{7}^{c} \\
a_{9}^{c} \\
\vdots
\end{array}\right]=\left[\begin{array}{c}
W_{0} \\
0 \\
0 \\
0 \\
0 \\
\vdots
\end{array}\right]} \\
{\left[\begin{array}{ccccccc}
K_{0}-L_{0} & L_{1} & 0 & 0 & 0 & \ldots & \\
L_{1} & K_{1} & L_{2} & 0 & 0 & 0 & \ldots \\
0 & L_{2} & K_{2} & L_{3} & 0 & 0 & \ldots \\
0 & 0 & L_{3} & K_{3} & L_{4} & 0 & \ldots \\
0 & 0 & 0 & L_{4} & K_{4} & L_{5} & \ldots \\
\vdots & \vdots & \vdots & \vdots & \vdots & \ddots &
\end{array}\right]\left[\begin{array}{c}
a_{1}^{s} \\
a_{3}^{s} \\
a_{5}^{s} \\
a_{7}^{s} \\
a_{9}^{s} \\
\vdots
\end{array}\right]=\left[\begin{array}{c}
0 \\
0 \\
0 \\
0 \\
0 \\
\vdots
\end{array}\right]}
\end{gathered}
$$


and

$$
\left[\begin{array}{ccccccc}
K_{1}^{\prime} & L_{2}^{\prime} & 0 & 0 & 0 & \ldots \\
L_{2}^{\prime} & K_{2}^{\prime} & L_{3}^{\prime} & 0 & 0 & 0 & \ldots \\
0 & L_{3}^{\prime} & K_{3}^{\prime} & L_{4}^{\prime} & 0 & 0 & \ldots \\
0 & 0 & L_{4}^{\prime} & K_{4}^{\prime} & L_{5}^{\prime} & 0 & \ldots \\
0 & 0 & 0 & L_{5}^{\prime} & K_{5}^{\prime} & L_{6}^{\prime} & \ldots \\
\vdots & \vdots & \vdots & \vdots & \vdots & \ddots &
\end{array}\right]\left[\begin{array}{c}
a_{2}^{s} \\
a_{4}^{s} \\
a_{6}^{s} \\
a_{8}^{s} \\
a_{10}^{s} \\
\vdots
\end{array}\right]=\left[\begin{array}{c}
0 \\
0 \\
0 \\
0 \\
0 \\
\vdots
\end{array}\right]
$$

where

$$
\begin{aligned}
L_{m}(k, \theta, \xi) & =\frac{3 \pi \Omega \sin ^{2} \theta}{2\left(1-\Omega^{2}\right)^{\frac{5}{2}}}+(\cos 2 \theta-1) F(m, \Omega), \\
K_{m}(k, \theta, \xi) & =\frac{\pi\left(2+\Omega^{2}-3 \Omega \cos ^{2} \theta\right)}{\left(1-\Omega^{2}\right)^{\frac{5}{2}}}-G(m+1, \Omega)+(1+\cos 2 \theta)(F(m, \Omega)+F(m+1, \Omega)),
\end{aligned}
$$

and

$$
\begin{aligned}
L_{m}^{\prime}(k, \theta, \xi) & =\frac{3 \pi \Omega \sin ^{2} \theta}{2\left(1-\Omega^{2}\right)^{\frac{5}{2}}}+\frac{1}{4}(\cos 2 \theta-1) G(m, \Omega) \\
K_{m}^{\prime}(k, \theta, \xi) & =\frac{\pi\left(2+\Omega^{2}-3 \Omega \cos ^{2} \theta\right)}{2\left(1-\Omega^{2}\right)^{\frac{5}{2}}}-4 F(m, \Omega)+\frac{1}{4}(1+\cos 2 \theta)(G(m, \Omega)+G(m+1, \Omega)) .
\end{aligned}
$$

By analogy with the above derivations, using Eq. (13), the recursive relations between the coefficients appeared in $b(\phi)$ are also obtained as

$$
\begin{aligned}
& \left(L_{0}+K_{0}\right) b_{1}^{c}+L_{1} b_{3}^{c}=0, \\
& L_{m} b_{2 m+1}^{c}+K_{m-1} b_{2 m-1}^{c}+L_{m-1} b_{2 m-3}^{c}=0, \quad m \geq 2 \\
& L_{1}^{\prime} b_{2}^{c}+K_{0}^{\prime} b_{0}^{c}=0, \\
& \left(L_{0}^{\prime}+L_{1}^{\prime} b_{0}^{c}+K_{1}^{\prime} b_{2}^{c}+L_{2}^{\prime} b_{4}^{c}=0,\right. \\
& L_{m}^{\prime} b_{2 m}^{c}+K_{m-1}^{\prime} b_{2 m-2}^{c}+L_{m-1}^{\prime} b_{2 m-4}^{c}=0, \quad m \geq 3 \\
& \left(K_{0}-L_{0}\right) b_{1}^{s}+L_{1} b_{3}^{s}=W_{0}, \\
& L_{m} b_{2 m+1}^{s}+K_{m-1} b_{2 m-1}^{s}+L_{m-1} b_{2 m-3}^{s}=0, \quad m \geq 2 \\
& K_{1}^{\prime} b_{2}^{s}+L_{2}^{\prime} b_{4}^{s}=0, \\
& L_{m}^{\prime} b_{2 m}^{s}+K_{m-1}^{\prime} b_{2 m-2}^{s}+L_{m-1}^{\prime} b_{2 m-4}^{s}=0 . \quad m \geq 3
\end{aligned}
$$

Solving the above recursive relations, results in the functions $a(\phi)$ and $b(\phi)$.

\section{Truncation}

In order to obtain the functions $a(\phi)$ and $b(\phi)$, we have to truncate the series in Eqs. (A1) and (A2) at some point. The accuracy of the results depends on the number of independent trigonometric functions we kept in the series. By keeping $l$ number of functions, the matrix equation in (A10) reduces to:

$$
\left[\begin{array}{cccccc}
L_{0}+K_{0} & L_{1} & 0 & 0 & \ldots & 0 \\
L_{1} & K_{1} & L_{2} & 0 & \ldots & 0 \\
0 & L_{2} & K_{2} & L_{3} & \ldots & \vdots \\
\vdots & \vdots & \vdots & \vdots & \vdots & 0 \\
0 & \ldots & 0 & L_{l-1} & K_{l-1} & L_{l} \\
0 & \ldots & 0 & 0 & L_{l} & K_{l}
\end{array}\right]\left[\begin{array}{c}
a_{1}^{c} \\
a_{3}^{c} \\
a_{5}^{c} \\
\vdots \\
a_{2 l-1}^{c} \\
a_{2 l+1}^{c}
\end{array}\right]=\left[\begin{array}{c}
W_{0} \\
0 \\
0 \\
\vdots \\
0 \\
0
\end{array}\right] .
$$



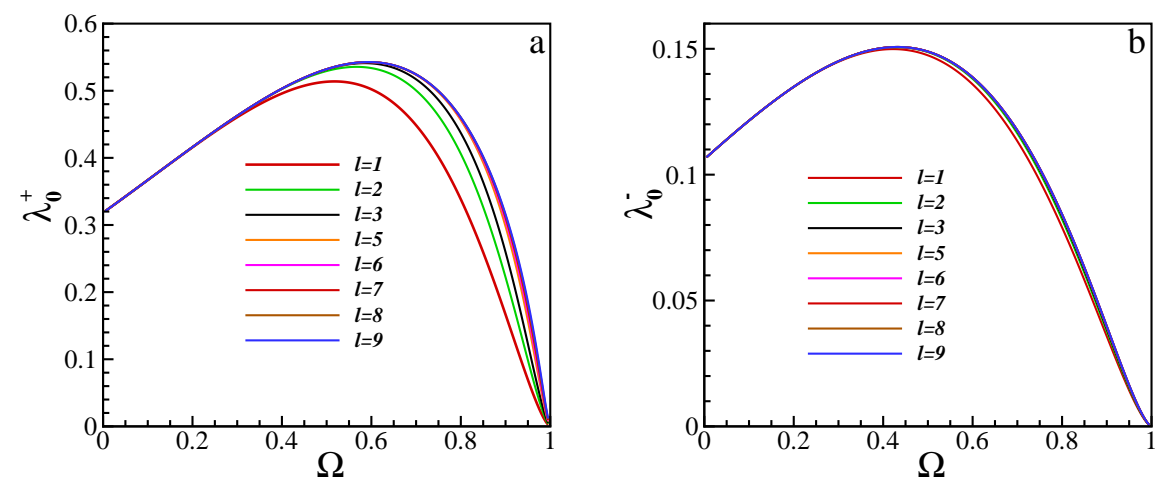

FIG. 12. (Color online) The functions $\lambda_{0}^{+}$(a) and $\lambda_{0}^{-}$(b) versus $\Omega$, for different values of $l$, in the case of highly anisotropic system $(\theta=\pi / 2)$.

The coefficient $a_{1}^{c}$ can be obtained from the relation $a_{1}^{c}=\operatorname{det} D_{1} / \operatorname{det} D$, where $D$ is the square matrix on the left hand side of Eq. (A18) and $D_{1}$ is a square matrix, obtaining from the $D$-matrix by replacing the first column with the column vector on the right hand side of the Eq. (A18). Computing the determinant of $D_{1}$ and $D$, the coefficient $a_{1}^{c}$ reads:

$$
a_{1}^{c}=\frac{W_{0}}{\left(L_{0}+K_{0}\right)-L_{1}^{2} \frac{d_{2}}{d_{1}}}=\frac{W_{0}}{\left(L_{0}+K_{0}\right)-\frac{L_{1}^{2}}{K_{1}-L_{2}^{2} \frac{d_{3}}{d_{2}}}}=\frac{W_{0}}{\left(L_{0}+K_{0}\right)-\frac{L_{1}^{2}}{K_{1}-\frac{L_{2}^{2}}{K_{2}-L_{3}^{2} \frac{d_{4}}{d_{3}}}}}=\ldots,
$$

where $d_{n}$ is the following determinant:

$$
d_{n}=\left|\begin{array}{ccccccc}
K_{n} & L_{n+1} & 0 & 0 & 0 & \ldots & 0 \\
L_{n+1} & K_{n+1} & L_{n+2} & 0 & 0 & \ldots & 0 \\
0 & L_{n+2} & K_{n+2} & L_{n+3} & 0 & \ldots & 0 \\
0 & 0 & \vdots & \vdots & \vdots & \ldots & 0 \\
\vdots & \vdots & \vdots & \vdots & \vdots & \vdots & 0 \\
\vdots & \vdots & \vdots & L_{l-1} & K_{l-1} & L_{l} & 0 \\
0 & 0 & 0 & \ldots & 0 & L_{l} & K_{l}
\end{array}\right|
$$

and $\frac{d_{n}}{d_{n-1}}=\left(K_{n-1}-L_{n}^{2} \frac{d_{n+1}}{d_{n}}\right)^{-1}$. By defining

$$
\lambda_{1}=\frac{L_{1}^{2}}{K_{1}-\frac{L_{2}^{2}}{K_{2}-\frac{L_{3}^{2}}{K_{3}-}}},
$$

the coefficient $a_{1}^{c}$ is written in the following compact form:

$$
a_{1}^{c}=\frac{W_{0}}{L_{0}+K_{0}-\lambda_{1}}=\tilde{\tau}_{k} \frac{\left(1+2 k^{2} \xi^{2}\right)^{3}}{k^{4} \xi^{4}} \lambda_{0}^{+} .
$$

where $\tilde{\tau}_{k}=\frac{\hbar^{2} v_{\mathrm{F}} A k^{3}}{\pi n_{c} J_{0}^{2} S^{2}}$ is a constant with a dimension of time. We have plotted in Fig. 12-a, the parameter $\lambda_{0}^{+}=$ $\left(K_{0}+L_{0}-\lambda_{1}\right)^{-1}$, versus $\Omega$, for different values of $l$. As it is shown, for $\Omega<0.5$ (i.e. for $k_{\mathrm{F}} \xi<1$ ) the function $\lambda_{0}^{+}$is independent of $l$ and a precise closed form expression for $\lambda_{0}^{+}$is obtained by keeping only $l=2$ number of independent 

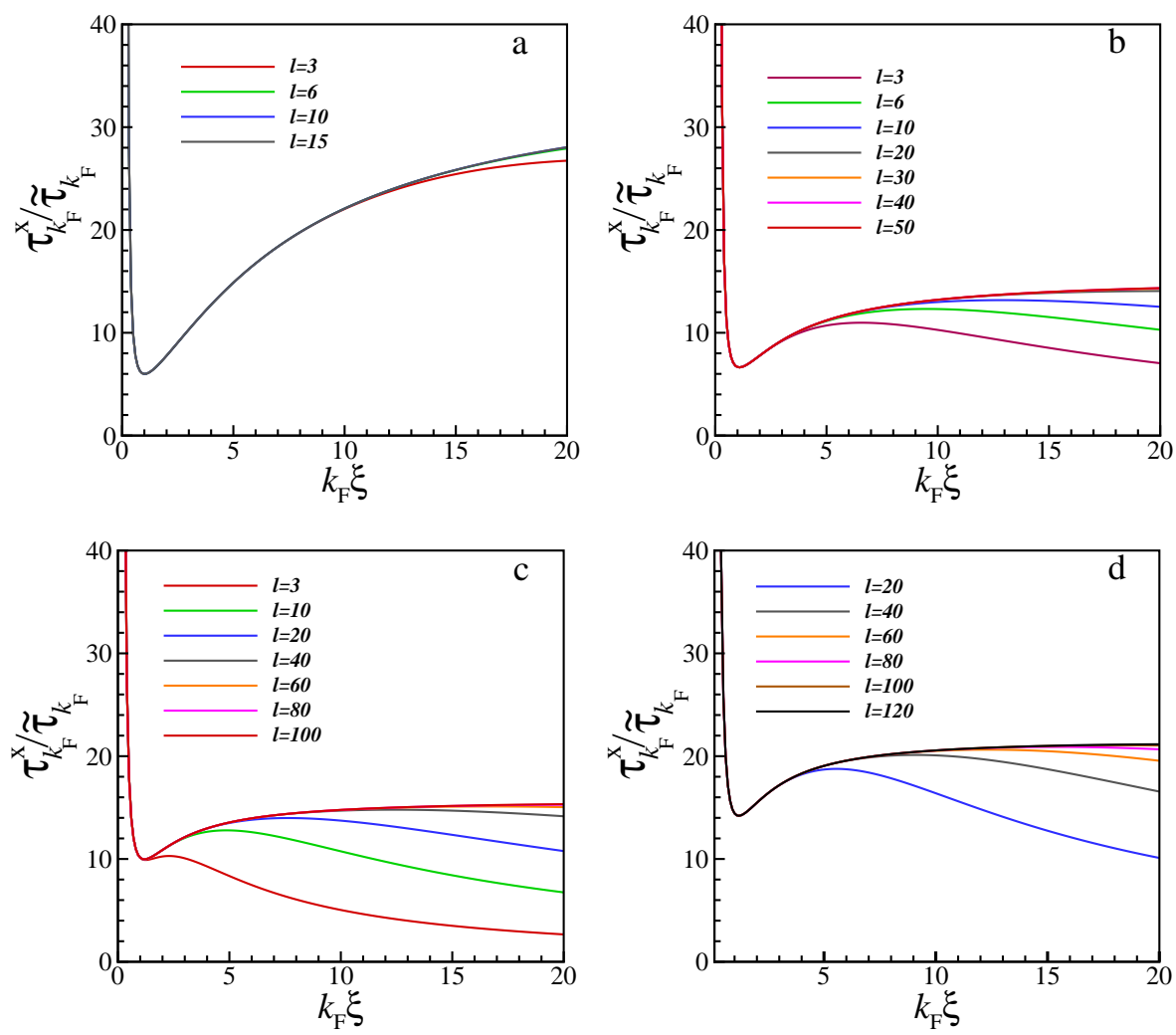

FIG. 13. (Color online) The dimensionless relaxation time $\tau_{k_{\mathrm{F}}}^{x} / \tilde{\tau}_{k_{\mathrm{F}}}$ versus $k_{\mathrm{F}} \xi$, for different values of $l$ at a) $\theta=10^{\circ}$, b) $\theta=30^{\circ}$, c) $\theta=60^{\circ}$ and d) $\theta=90^{\circ}$. By increasing $\theta$, the TI becomes more anisotropic and we need more independent functions to reach to a precise closed form expression for $\tau_{k}^{x}$.

trigonometric functions in the series. For $\Omega>0.5$ or $k_{\mathrm{F}} \xi>1$, the situation is however different and the number $l$ is crucial to reach to the precise value of $\lambda_{0}^{+}$. For an arbitrary $\Omega(>0.5)$, the parameter $\lambda_{0}^{+}$increases by increasing $l$ and saturates rapidly at a finite $l$. Thus by employing a finite number of independent functions (for example $l \sim 9$ ), we obtain a closed form expression for $\lambda_{0}^{+}$.

As we have explained in section IV,$a_{1}^{c}$ is indeed the effective relaxation time $\tau_{k}^{x}$, appearing in the relation of the surface conductivity $\sigma_{x x}$. We have plotted in Fig. 13, the dimensionless relaxation time $\tau_{k_{\mathrm{F}}}^{x} / \tilde{\tau}_{k_{\mathrm{F}}}$ versus $k_{\mathrm{F}} \xi$, for different values of $l$ and for tilt angles $\theta=10^{\circ}, 30^{\circ}, 60^{\circ}$ and $90^{\circ}$. Independent of $\theta$, similar to the parameter $\lambda_{0}^{+}$, for $k_{\mathrm{F}} \xi<1$, the relaxation time $\tau_{k_{\mathrm{F}}}^{x}$ does not depend on $l$ and employing a few number of independent functions gives the precise value of $\tau_{k_{\mathrm{F}}}^{x}$. For $k_{\mathrm{F}} \xi>1$, effects of anisotropy emerge and depending on the tilt angle $\theta$, a large $l$ is required to reach a precise closed form expression for $\tau_{k}^{x}$. When the tilt angle increases from 0 to $90^{\circ}$, the TI becomes more anisotropic and the relaxation time versus $l$ saturates at larger values of $l$. For example, at $\theta=10^{\circ}$, for $k_{\mathrm{F}} \xi \gg 1$, the relaxation time $\tau_{k}^{x}$ saturates at $l \sim 15$, however at $\theta=90^{\circ}$, we need to employ $l \sim 120$ independent functions, to attain a precise expression for $\tau_{k}^{x}$.

The other coefficients $a_{2 n+1}^{c}$ can also be obtained in terms of $a_{1}^{c}$, by using the recursive relations in (19).

The number of Dirac electrons should be conserved in scattering. This implies that the coefficient $a_{0}^{c}$ and consequently all $a_{2 n}^{c}$ should be zero. Furthermore, since the linear equations obtained from the recursive relations in (23) are homogeneous, all the coefficients $a_{n}^{s}$ with odd and even indices are zero. The function $a(\phi)$ is finally given by

$$
a(\phi)=\sum_{m=0}^{l} a_{2 m+1}^{c} \cos (2 m+1) \phi
$$

By analogy with the derivation of $a(\phi)$, we can show that the recursive relations in (A15), (A16) and (A17) lead to the coefficient $b_{1}^{s}$ :

$$
b_{1}^{s}=\frac{W_{0}}{K_{0}-L_{0}-\lambda_{1}}=\lambda_{0}^{-} W_{0}
$$


where $\lambda_{0}^{-}=\left(K_{0}-L_{0}-\lambda_{1}\right)^{-1}$. We have plotted in Fig. 12-b, the function $\lambda_{0}^{-}$versus $\Omega$ for different values of the truncation parameter $l$. As it is shown, for small values of $k_{\mathrm{F}} \xi$, we can obtain a precise value for $\lambda_{0}^{-}$, by keeping only a few number of independent functions (for example $l \simeq 3$ ). The other $b_{2 n+1}^{s}$ coefficients can be obtained by the recursive relations in (A17). Particle number conservation again dictates that $b_{0}$ should be zero which results in $b_{2 n}^{s}=0$. The coefficients of the cosine functions are also zero because the linear equations obtained from the recursive relation (A15) and (A16) are homogeneous. Finally the function $b(\phi)$ is given by

$$
b(\phi)=\sum_{m=0}^{l} b_{2 m+1}^{s} \sin (2 m+1) \phi .
$$

* jahan@iasbs.ac.ir

1 C. L. Kane and E. J. Mele, Phys. Rev. Lett. 95, 146802 (2005).

2 B. A. Bernevig, T. L. Hughes, and S. Zhang, Science 314, 1757 (2006).

3 J. E. Moore and L. Balents, Phys. Rev. B 75, 121306 (2007).

${ }^{4} \mathrm{~L} . \mathrm{Fu}, \mathrm{C} . \mathrm{L}$ Kane, and E. J. Mele, Phys. Rev. Lett. 98, 106803 (2007).

${ }^{5}$ M. König, S. Wiedmann, C. Brüne, A. Roth, H. Buhmann, L. W. Molenkamp, X.-L. Qi, and S.-C. Zhang, Science 318, 766 (2007).

6 D. Hsieh, D. Qian, L. Wray, Y. Xia, Y. S. Hor, R. J. Cava, and M. Z. Hasan, Nature 452, 970 (2008).

7 S.-Q. Shen, Topological Insulators: Dirac Equation in Condensed Matters (Berlin: Springer, 2012).

8 C. L. Kane and E. J. Mele, Phys. Rev. Lett. 95, 226801 (2005).

9 B. A. Bernevig and S.-C. Zhang, Phys. Rev. Lett. 96, 106802 (2006).

${ }^{10}$ L. Fu and C. L. Kane, Phys. Rev. B 76, 045302 (2007).

${ }^{11} \mathrm{~L}$. $\mathrm{Fu}$ and $\mathrm{C}$. L. Kane, Phys. Rev. Lett. 102, 216403 (2009).

12 X.-L. Qi, T. L. Hughes, and S.-C. Zhang, Phys. Rev. B 78, 195424 (2008).

13 R. Yu, W. Zhang, H.-J. Zhang, S.-C. Zhang, X. Dai, and Z. Fang, Science 329, 61 (2010).

14 D. Culcer, E. H. Hwang, T. D. Stanescu, and S. Das Sarma, Phys. Rev. B 82, 155457 (2010).

15 D. Culcer, Physica E 44, 860 (2012).

16 L. A. Wray, S.-Y. Xu, Y. Xia, D. Hsieh, A. V. Fedorov, Y. S. Hor, R. J. Cava, A. Bansil, H. Lin, and M. Z. Hasan, Nature Physics 7, 32 (2011).

17 Y. L. Chen, J.-H. Chu, J. G. Analytis, Z. K. Liu, K. Igarashi, H.-H. Kuo, X. L. Qi, S. K. Mo, R. G. Moore, D. H. Lu, M. Hashimoto, T. Sasagawa, S. C. Zhang, I. R. Fisher, Z. Hussain, and Z. X. Shen, Science 329, 659 (2010).

18 X. F. Kou, W. J. Jiang, M. R. Lang, F. X. Xiu, L. He, Y. Wang, Y. Wang, X. X. Yu, A. V. Fedorov, P. Zhang, and K. L. Wang, Journal of Applied Physics 112, 063912 (2012).

19 D. Culcer and S. Das Sarma,
Phys. Rev. B 83, 245441 (2011).

20 A. A. Burkov and D. G. Hawthorn, Phys. Rev. Lett. 105, 066802 (2010).

21 A. Sabzalipour, J. Abouie, and S. H. Abedinpour, Journal of Physics: Condensed Matter. 27, 115301 (2015).

22 Z.-G. Fu, P. Zhang, Z. Wang, F. Zheng, and S.-S. Li, Phys. Rev. B 85, 245433 (2012).

23 K. Akabli, Y. Magnin, M. Oko, I. Harada, and H. T. Diep, Phys. Rev. B 84, 024428 (2011).

${ }^{24} \mathrm{~K}$. Akabli and H. T. Diep, Phys. Rev. B 77, 165433 (2008).

${ }^{25}$ K. Výborný, A. A. Kovalev, J. Sinova, and T. Jungwirth, Phys. Rev. B 79, 045427 (2009).

26 A. Kandala, A. Richardella, S. Kempinger, C.-X. Liu, and N. Samarth, Nature Communications 6, 7434 (2015).

27 Y. A. Bychkov and R. E. I., JET Letters 39, 76 (1984).

28 H. Zhang, C.-X. Liu, X.-L. Qi, X. Dai, Z. Fang, and S.-C. Zhang, Nature Physics 5, 438 (2009).

${ }^{29}$ K. Christensen and N. R. Moloney, Complexity and Criticality (Imperial College London, UK, 2005).

30 A. Coniglio and W. Klein, J. Phys. A13, 2775 (1980).

31 J. Hoshen and R. Kopelman, Phys. Rev. B 14, 3438 (1976).

32 N. W. Ashcroft and N. D. Mermin, Solid State Physics (Harcourt College Publishers, 1975).

33 J. Schliemann and D. Loss, Phys. Rev. B 68, 165311 (2003).

34 A. W. Rushforth, K. Výborný, C. S. King, K. W. Edmonds, R. P. Campion, C. T. Foxon, J. Wunderlich, A. C. Irvine, P. Vavsek, V. Novák, K. Olejník, J. Sinova, T. Jungwirth, and B. L. Gallagher, Phys. Rev. Lett. 99, 147207 (2007).

35 M. Trushin and J. Schliemann, Phys. Rev. B 75, 155323 (2007).

36 G. Rosenberg and M. Franz, Phys. Rev. B 85, 195119 (2012).

37 S. Zhang and Z. Li, Phys. Rev. Lett. 93, 127204 (2004).

38 Y. Araki and K. Nomura, Phys. Rev. B 96, 165303 (2017).

39 D. Andrikopoulos and B. Sorée, Scientific Reports 7, 17871 (2017).

40 N. A. Sinitsyn, A. H. MacDonald, T. Jungwirth, V. K. Dugaev, and J. Sinova, Phys. Rev. B 75, 045315 (2007). 